\title{
1 Cytoskeletal regulation of a transcription factor by DNA mimicry
}

3 Farah Haque ${ }^{1,2}$, Christian Freniere ${ }^{1,2}$, Qiong $\mathrm{Ye}^{1,2}$, Nandini Mani ${ }^{1,2, \dagger}$, Elizabeth M. Wilson-

4 Kubalek $^{3, \dagger}$, Pei-I Ku ${ }^{1,2}$, Ronald A. Milligan ${ }^{3}$ and Radhika Subramanian ${ }^{1,2, *}$

$6 \quad{ }^{1}$ Department of Molecular Biology, Massachusetts General Hospital, Boston, MA 02114, USA

$7 \quad{ }^{2}$ Department of Genetics, Harvard Medical School, Boston, MA 02115, USA

$8{ }^{3}$ Department of Integrative Structural and Computational Biology, Scripps Research, La Jolla, CA

992037, USA

10 *Correspondence: radhika@molbio.mgh.harvard.edu

11 †These authors contributed equally 


\section{Abstract}

13 A long-established strategy for transcription regulation is the tethering of transcription

14 factors to cellular membranes. In contrast, the principal effectors of Hedgehog signaling, the Gli

15 transcription factors, are regulated by microtubules in the primary cilium and the cytoplasm. How

16 Gli is tethered to microtubules remains unclear. We uncover DNA mimicry by the ciliary kinesin

17 Kif7 as a mechanism for the recruitment of Gli to microtubules, revealing a new mode of tethering

18 a DNA-binding protein to the cytoskeleton. Gli increases the Kif7-microtubule affinity and

19 consequently modulates the localization of both proteins to microtubules and the cilium tip. Thus,

20 the kinesin-microtubule system is not a passive Gli tether but a regulatable platform tuned by the

21 kinesin-transcription factor interaction. We re-tooled the unique DNA-mimicry-based Gli-Kif7

22 interaction for inhibiting the nuclear and cilium localization of Gli. This strategy can be potentially

23 exploited for downregulating erroneously activated Gli in human cancers. 


\section{Main text}

25 The tethering of transcription factors to membranes of cytoplasmic organelles (e.g. the

26 endoplasmic reticulum, Golgi and mitochondria) or the plasma membrane, and their signal-

27 dependent trafficking into the nucleus is a well-established mechanism for the precise regulation

28 of gene repression and activation ${ }^{1}$. In recent years, the microtubule cytoskeleton has emerged as

29 an important scaffold for tethering transcription factors away from the nucleus for pathway

30 repression ${ }^{2-4}$. However, the molecular and structural mechanisms by which the cytoskeleton

31 regulates transcription factors remains poorly understood.

An essential developmental pathway with a strict and conserved requirement for the

33 microtubule cytoskeleton is Hedgehog $(\mathrm{Hh})$ signaling $^{5-8}$. In vertebrates, this pathway has a unique

34 dependence on the primary cilium ${ }^{9}$, a microtubule-based organelle. Central to regulation of the

35 vertebrate $\mathrm{Hh}$ signaling is the trafficking and accumulation of Gli proteins (Ci; Drosophila

36 Melanogaster homolog) in the primary cilium. The Gli family of proteins consist of three isoforms

37 Gli1, Gli2 and Gli3. Of these proteins, Gli1 is a transcriptional activator ${ }^{10}$, and Gli2 and Gli3 are

38 dual-transcription factors characterized by the following domain organization: a repressor domain

39 at the N-terminus and a trans-activator domain at the C-terminus that are separated by DNA-

40 binding zinc-finger domain and sites for proteolytic cleavage ${ }^{11,12}$. During Hh pathway repression,

41 Gli2 and Gli3 are proteolytically processed into the shorter repressor form at the base of the cilium,

42 with Gli3 acting as the major repressor ${ }^{13-15}$. Activation of the pathway results in increased

43 accumulation of the full-length activator forms of the Gli proteins at the distal cilium tip, with Gli2

44 acting as the major activator, which promotes the expression of Gli1 ${ }^{16-19}$. In vertebrates, defects in

45 cilium assembly and architecture, result in incorrect processing of the Gli proteins ${ }^{20,21}$. These

46 observations suggest that the microtubule cytoskeleton plays an active role in Hh pathway 
47 activation. However, the mechanism of microtubule-dependent Gli regulation remains poorly

48 understood.

The non-motile ciliary kinesin, Kif7 (ortholog of the Drosophila melanogaster Costal-2

50 kinesin-like protein and homolog of the motile ciliary kinesin Kif27), is a core Hh pathway

51 component that provides the molecular link between the microtubule cytoskeleton and Gli

52 regulation ${ }^{22-25}$. Loss of function mutations of Kif7 in zebrafish and mice embryos show defects in

53 Hh-dependent developmental patterning ${ }^{22-24,26}$, and clinical mutations in Kif7 have been associated

54 with severe ciliopathies ${ }^{27,28}$. When the Hh pathway is off, Kif7 is largely present at the base of the

55 cilium, where it is thought to promote the formation of the repressor form of Gli. Upon pathway

56 activation, both Kif7 and Gli accumulate at the tip of the primary cilium, a step required for Gli

57 activation ${ }^{25}$. The precise mechanism for the localization of Kif7 to the base of the cilia is not known

58 and multiple mechanisms have been suggested for its trafficking and cilium tip localization ${ }^{25,29-33}$.

59 In the absence of Kif7, Gli can enter the cilium but is not correctly concentrated at the distal tip of

60 the axoneme ${ }^{30}$. These observations together with the reported co-precipitation of Gli proteins with

61 Kif7 in cell and embryo lysates ${ }^{23}$ have led to the proposal that microtubule-bound Kif7 establishes

62 a 'cilium-tip compartment', a passive platform at the distal cilium tip, to which Gli is recruited ${ }^{25,30}$.

63 However, although current models assume a direct binding between Gli and Kif7, this has not been

64 conclusively established and the molecular and structural basis of their interaction remains elusive.

65 Furthermore, it is unknown whether the Kif7-Gli interaction is sufficient to tether the transcription

66 factor to microtubules, and how the Kif7-Gli interaction contributes to the organization of the

67 cilium tip compartment and Gli regulation. The lack of mechanistic insights has precluded a deeper

68 understanding of the role of the microtubule cytoskeleton and the primary cilium in Hh signaling.

69 Here we address these fundamental questions and uncover new structural and biochemical design 
bioRxiv preprint doi: https://doi.org/10.1101/2021.09.30.462597; this version posted October 1, 2021. The copyright holder for this preprint

(which was not certified by peer review) is the author/funder, who has granted bioRxiv a license to display the preprint in perpetuity. It is made available under aCC-BY-NC-ND 4.0 International license.

70 principles by which the cytoskeleton can act as a cytoplasmic scaffold for transcription factor

71 regulation. 


\section{Results}

73 DNA structural mimicry underlies Gli transcription factor binding to Kif7

Gli proteins were shown to co-immunoprecipitate with Kif7 in pull-down assays from

75 mouse embryo lysates ${ }^{23}$. However, biochemical and structural characterization of Gli-Kif7 complexes has been challenging due to high proteolytic sensitivity and low solubility of these large

77 (>150 kDa) multi-domain proteins. To overcome this technical challenge, we used a pull-down 78 assay to map the interaction domains between Gli and Kif7. We observed that the DNA-binding

79 zinc finger domain of Gli2 (418-594aa; Gli2-ZF) bound to the first coiled-coil dimerization domain

80 of Kif7 (460-600aa; Kif7-CC) (Fig. 1a,b). In addition, a weak signal corresponding to interaction

81 between monomeric Kif7 motor domain (1-361 aa) and Gli2-ZF was observed (Extended Fig 1a).

We first characterized the interaction between Kif7-CC and Gli2-ZF. Bio-layer

83 interferometry (BLI)-based binding assay with purified recombinant proteins (Extended Data

84 Fig. 1b,c) showed tight binding of the proteins in the Kif7-CC:Gli2-ZF complex with a $\mathrm{K}_{\mathrm{d}}$ of 48

$85 \pm 5 \mathrm{nM}$ (Fig. 1c). We were able to further narrow the interaction site on Kif7 to a 63 amino acid

86 shorter coiled-coil segment of Kif7-CC (481-543aa; Kif7-SCC) that binds Gli2-ZF with a similar

$87 \mathrm{~K}_{\mathrm{d}}$ of $69 \pm 20 \mathrm{nM}$ (Fig. 1c). Together, these data show that Kif7 uses its coiled-coil dimerization

88 domain, and not the canonical C-terminal 'cargo' binding domain in kinesins, to bind Gli2 with

89 high affinity, while Gli2 uses its DNA-binding domain to bind Kif7 dimer. Thus, the domain of

90 the Gli transcription factor that mediates its interaction with DNA in the nucleus is repurposed to

91 interact with a kinesin in the cytoplasm.

92 How does the DNA binding domain of Gli bind the coiled-coil dimerization domain of

93 Kif7? Single molecule fluorescence intensity and photobleaching analysis of the GFP-tagged

94 proteins indicate that the GST-Kif7-CC is an obligate dimer and Gli2-ZF is a monomer (Extended 
95 Data Fig. 1d,e). We determined that the stoichiometry of the Kif7-CC-GFP:Gli2-ZF-GFP

96 complex was 2:1; using in-gel GFP fluorescence and quantitative Western blot (Fig. 1d and

97 Extended Data Fig. 1f,g; see Methods). Thus, one molecule of monomeric Gli2-ZF binds to one

98 Kif7-CC dimer.

To gain insight into the structural basis of the Kif7-SCC:Gli2-ZF complex, we performed

100 comparative homology modeling. The structure of Gli2-ZF domain was modelled using the X-ray

101 structure of the zinc-finger domain of Gli1 isoform as a template (PDB: 2GLI, >95\% sequence

102 similarity) ${ }^{34}$ (Fig. 2a). The Kif7 coiled-coil domain was modeled as a homodimer with high

103 accuracy using X-ray structures of other coiled-coil domains as template (Fig. 2c). The complex

104 between Gli and DNA is characterized by the positively charged zinc-finger domain of Gli

105 wrapping around the major groove of the rod-like DNA double-helix. Extensive electrostatic

106 contacts exist between the negatively charged phosphate backbone of DNA and the second and

107 third zinc-fingers in $\mathrm{Gli}^{34}$. As expected, the structural model of Gli2-ZF is very similar to Gli1-ZF

108 (Extended Fig. 2a-c and Fig 2b). Analysis of the structural model of Kif7-SCC revealed two

109 striking features. First, the overall size and shape of Kif7-SCC is similar to dsDNA. Second,

110 analysis of the electrostatic surface potential of Kif7-SCC ( $\mathrm{pI}=4.48)$ shows a highly negatively

111 charged rod-shaped surface, similar to the DNA backbone (Fig. 2c). Modelling the structure of the

112 Kif7-Gli2 complex indicates that the positively charged interface of Gli2 zinc-fingers clamp the

113 negatively charged Kif7-SCC rod-like domain (Extended Data Fig. 2a-c) in a manner similar to

114 the Gli-DNA complex. The stark similarities in electrostatic surface charge complementarity,

115 molecular dimensions, and mode of binding of Gli2-ZF with DNA and Kif7-SCC suggests that

116 Kif7 may be a DNA molecular mimic for Gli binding in the cytoplasm. 
To test the structural model of the Kif7-CC:Gli2-ZF interaction we first examined whether

118 Kif7-CC and DNA compete for the same binding site on Gli2. We measured the binding between

119 Gli2-ZF and Kif7-CC in the presence of a Gli-specific target dsDNA (TRE-2S) ${ }^{35}$. In the presence

120 of $125 \mathrm{nM}$ TRE-2S DNA there was no detectable binding between Kif7-CC and Gli2-ZF until the

121 analyte concentration was above 125nM Gli2-ZF (Fig. 2d). This suggests that DNA-bound Gli2-

122 ZF cannot bind Kif7-CC and that these interactions are mutually exclusive. We conducted the BLI

123 binding assay in buffers with increasing ionic strength and observed a systematic reduction in the

124 measured binding response from Kif7-CC and Gli2-ZF interaction, confirming that the interaction

125 between these proteins is driven by electrostatic interactions (Fig. 2e).

Finally, we performed a series of site-directed mutagenesis experiments to further confirm

127 the structural model and map the residues of Gli2-ZF and Kif7-CC required for complex formation.

128 BLI binding assay with Gli2-ZF truncations containing different groups of zinc-fingers indicated

129 the necessary requirement for zinc-fingers 2 and 3 in tandem for Kif7-CC binding (Extended Data

130 Fig. 3a-d). This is similar to the Gli-DNA interaction where ZF2 and ZF3 are critical for making

131 contacts with the phosphate backbone of $\mathrm{DNA}^{34}$. Mutation of specific charged residues in Gli2

132 ZF-2 (H493A/R496A) and ZF-3 (H503A/R516A/K521A) abolished binding to Kif7-CC whereas

133 those in ZF-4 (R550A/K552A/R556A) did not affect binding (Extended Data Fig. 3e,f).

134 Interestingly, the residues H493 (ZF2), R516 (ZF3) and K512 (ZF3) also engage in phosphate

135 backbone contacts with $\mathrm{DNA}^{34}$, suggesting that the same subset of amino acids interact with Kif7-

136 CC. Analysis of the interaction surface on Kif7-CC revealed three patches of negatively charged

137 amino acid residues (labeled as S1, S2 and S3 in Extended Data Fig. 3f) that are potential Gli2-

138 ZF binding residues. Systematic mutation of residues in these patches revealed that while Kif7-

$139 \mathrm{CC}$ S1 (E500A/E501A/E502A/D505A) mutant abolished binding to Gli2-ZF, the S2 
141 3e,f). To further validate the interaction, we expressed and purified four recombinant S1 mutants

142 (D505A, E501A/E502A, E502A, E502A/D505A). All four mutants elute similar to wild type

143 protein in size exclusion chromatography, indicating that they are properly folded (Extended Data

144 Fig. 3g,h). Three of these mutants (E501A/E502A, E502A, E502A/D505A) showed no binding to

145 Gli2-ZF while the fourth one (D505A), shows diminished binding (Extended Data Fig. 3i). This

146 suggests that negatively charged residues included in S1 that lie approximately at the center of the

147 coiled-coil rod of Kif7 form the primary contact point for Gli binding.

148 Taken together, the results from DNA competition assay, ionic strength-dependence and

149 the mutagenesis studies provide additional support for DNA mimicry by the coiled-coil domain of

150 Kif7. The center of this negatively-charged rod forms the Gli2 interaction site with zinc-fingers 2

151 and 3 making key charge-charge contacts, as seen previously in binding of Gli to DNA ${ }^{34}$.

\section{Second Gli binding site on the Kif7 motor domain}

We characterized the interaction between Gli2-ZF and the dimeric Kif7. We determined

155 that the stoichiometry of Kif7-DM:Gli2-ZF complex in the absence of microtubules was 1:1,

156 suggesting there are two molecules of Gli2-ZF bound per Kif7-DM (Fig. 3a and Extended Data

157 Fig. 4a-e). Since one Gli2-ZF is bound to the coiled-coil dimer of Kif7, we hypothesized that the

158 second Gli2-ZF molecule binds proximal to the Kif7 motor domains, as was also suggested by the

159 weak signal observed in the pull-down assay using cell lysate (Extended Data Fig. 1a). However,

160 we failed to detect any binding between the purified recombinant monomeric Kif7 motor domain

161 (1-386aa, Kif7-MM) and Gli2-ZF at concentrations as high as $12 \mu \mathrm{M}$ Gli2-ZF in the BLI assay.

162 The differences in the two experiments could arise from the presence of low levels of intact 
163 microtubules in the cell extracts. We therefore examined the recruitment of Gli to GMPCPP-

164 polymerized microtubules by Kif7 using an in vitro TIRF microscopy assay (Fig. 3b). We

165 examined the microtubule localization of Alexa-647 labeled Gli2-ZF-SNAP in the presence of a

166 monomeric Kif7 motor domain construct (1-386aa, Kif7-MM-GFP), which lacks the coiled-coil

167 Gli interaction site, and compared it to dimeric Kif7 protein (1-543aa, Kif7-DM-GFP), which has

168 both sites. Analysis of the Alexa-647 intensity reveals microtubule-bound Kif7-MM can bind Gli2-

$169 \mathrm{ZF}$, albeit to a lesser extent when compared to the Kif7-DM ( $\sim 9$ fold versus $\sim 3$ fold above the

170 background fluorescence of Gli alone on microtubules respectively) (Fig. 3c). These data suggest

171 that the motor domain of Kif7 likely forms a Gli-interaction site, but this interaction requires

172 concentrating motors on microtubules or another mechanism, such as dimerization, that brings the

173 motor domains in proximity.

174 To visualize the binding of Gli2-ZF to the Kif7 motor domain we obtained cryo-EM

175 reconstructions of Kif7-DM bound to microtubules in the presence of Gli2-ZF-SNAP and the non-

176 hydrolysable ATP analogue, AMP-PNP (3.9 Å resolution, Fig. 3d, Extended Data Fig. 5a,b and

177 Extended Data Table 1). Comparisons of the cryo-EM reconstruction with a map of microtubule-

178 bound Kif7 motor domain in the AMP-PNP state (PDB:6MLR, EMD:9141) showed that the motor

179 domain adopts the same conformation while binding to microtubules in the presence and absence

180 of Gli2-ZF (green in Fig. 3d). While no additional density was seen bound to the microtubule

181 lattice, an extra region of density contacting the motor domain of Kif7 was observed in the

182 presence of Gli2-ZF-SNAP (pink in Fig. 3d). Close inspection reveals that this extra density lies

183 close to loop L6, helices $\alpha-2$ and $\alpha-3$ on the Kif7 motor domain and away from the nucleotide-

184 binding pocket as well as the microtubule-binding interface of the motor (Supplementary Video

185 1). We confirmed that the extra density originated from the Gli2-ZF protein by generating and 
193 of the cryo-EM maps obtained in the presence of Gli2-ZF and Gli2-ZF-SNAP was used to 194 unambiguously assign the density corresponding to Gli2-ZF (Extended Data Fig. 5d). We built a

195 structural model of the Gli2-ZF peptide backbone sequence (see Methods) into this extra density 196 and were able to fit two complete and one partial zinc-fingers (Extended Data Fig. 5e and

197 Supplementary Video 1). Since it is well established that under saturating motor concentrations 198 needed for cryo-EM reconstructions, the density of only a single motor head can be resolved ${ }^{36,37}$, 199 we do not expect to see the entire Gli2-ZF interaction site formed by both motor heads and the 200 coiled-coil domain in a dimer. TIRFM assays with Alexa-647 tagged Gli2-ZF1-3-SNAP protein 201 show that first three ZFs of Gli are sufficient to bind Kif7-MM (Extended Data Fig. 4f). Together 202 these results confirm that in addition to the high affinity interaction site on Kif7-CC, there is a 203 second interaction site for Gli2-ZF proximal to the Kif7 motor domain.

204 Kif27 is a close homolog of Kif7 and these two proteins are thought to arise from a gene 205 duplication event ${ }^{38,39}$. Unlike Kif7, Kif27 plays a role in motile cilium biogenesis and is not 206 thought to be involved in Hedgehog signaling ${ }^{40}$. We wondered if the structural determinants of Gli 207 interaction sites are conserved or dissimilar between Kif7 and Kif27. We modelled the motor 208 domain (1-398aa) and the coiled-coil dimerization domains (480-550aa) of Kif27 and Kif7 and 
compared their electrostatic surface potential. Our analysis revealed that the electrostatic surface

210 potential of Kif27-CC is overall neutral in contrast to Kif7-CC, and the motor domain regions of

211 Kif27 (helix $\alpha-2, \alpha-3$ and loop L6) corresponding to the Gli2-ZF interaction site in Kif7, present

212 a less negative surface potential (Fig. 3e). Consistent with this, we do not observe recruitment of

213 Gli2-ZF on microtubules by the dimeric Kif27-DM-GFP (1-580aa) protein in our TIRF-

214 microscopy reconstitution assay (Fig. 3c). The comparative structural analysis of Kif7 and Kif27

215 indicates a specific adaptation in Kif7 for Gli binding and Hedgehog signaling.

217 Synergistic localization of Kif7 and Gli to microtubules in vitro

218 We next examined if the binding of Gli altered the microtubule-binding of Kif7. The

219 microtubule binding of Kif7-DM-GFP was imaged in the absence or presence of Alexa-647

220 labeled Gli2-100nM ZF-SNAP in a TIRFM assay. As expected, analysis of Alexa-647 intensity

221 showed 3-fold increase in recruitment on microtubules in the presence of Kif7-DM (Fig. 4a). Low

222 levels of Gli2 fluorescence intensity were visualized on microtubules even in the absence of Kif7,

223 which occurs from non-specific electrostatic interactions in lower ionic strength BRB80 buffer.

224 Interestingly, analysis of Kif7 fluorescence intensity $(100 \mathrm{nM})$ revealed a $>5$-fold increase in Kif7

225 binding to microtubules in the presence of Gli2-ZF (50nM) (Fig. 4b). Thus, Gli binding to Kif7

226 changes the microtubule binding affinity of Kif7 and sets up a positive feedback loop that in turn

227 results in enhanced recruitment of Gli to microtubules. This positive feedback in the kinesin-

228 microtubule binding generates a graded response that is sensitive to increasing Gli concentrations

229 until it reaches saturation (Fig. 4c). Further examination of the Kif7-microtubule interaction

230 revealed an increase in the residence time of single molecules of Kif7-DM-GFP on microtubules

231 with increasing concentrations of Gli2-ZF. (Extended Data Fig. 6a-c). We validated the findings 
232 from TIRF assays using a co-sedimentation assay, which showed that Kif7 has a higher

233 microtubule binding affinity in the presence of Gli2-ZF $\left(\mathrm{K}_{\mathrm{d}}=0.54 \pm 0.03 \mu \mathrm{M}\right)$ compared to when

234 Gli is absent $\left(\mathrm{K}_{\mathrm{d}}=4.23 \pm 0.24 \mu \mathrm{M}\right)$ (Fig. 4d and Extended Data Fig. 6d).

235 We wanted to determine the contribution of the two Gli interaction sites on Kif7 in

236 regulating the Kif7-microtubule interaction. We first hypothesized that the binding of Gli2-ZF to

237 the Kif7 motor domain may underlie the observed changes in the Kif7-microtubule binding.

238 Therefore, we generated a chimera in which the Kif7 motor domains are intact, but the Kif7-CC

239 is replaced by Kif27-CC (Kif7M-Kif27CC) (Fig. 4e and Extended Data Fig. 7a,b). To our

240 surprise the Kif7M-Kif27CC chimera has constitutive higher binding to microtubules compared

241 to Kif7-DM and the kinesin-microtubule interaction was independent of Gli concentration (Fig.

242 4e). To test this further we generated a point mutation in Kif7-DM (E502A), which eliminates Gli

243 binding to the coiled-coil domain of Kif7 (Extended Data Fig. 3e). Similar to Kif7M-Kif27CC,

244 Kif7-DM(E502A) is a constitutive dimer and recruits lower levels of Gli2-ZF relative to wild type

245 as expected for constructs lacking one Gli binding site (Extended Data Fig. 7). We find that this

246 mutant is identical to the Kif7M-Kif27CC chimera in terms of Gli-independent strong microtubule

247 binding (Fig.4e). Thus, counterintuitive to our expectation, the binding of Gli2-ZF to the Kif7-CC

248 domain rather than the Kif7 motor domain has a dominant role in the regulation of Kif7-

249 microtubule interaction. These data are consistent with a mechanism where the coiled-coil domain

250 inhibits the Kif7-microtubule binding, and this inhibition is relieved by Gli binding to the coiled-

251 coil and motor domains.

252 To test if the inhibition by the coiled-coil is specific to the Kif7 motor domain, we 253 generated a chimera where the motor and neck linker domain of Kif7 was replaced by that of Kif27

254 (Kif27M-Kif7CC). Similar to the other mutants described in this section, this chimera is dimeric 
and recruits lower levels of Gli compared to wild type Kif7-DM (Extended Data Fig. 7). We

256 found that the microtubule binding of Kif27M-Kif7CC chimera exhibited lower dependence on

257 Gli concentrations (Fig. 4e and Extended Data Fig. 7). Together these data suggest that the

258 inhibition of the Kif7-microtubule binding by the Kif7 coiled-coil domain is highest in the context

259 of the Kif7 motor domains in the Kif7-DM, and the complete alleviation of this inhibition by Gli

260 sets the dynamic range of the Kif7-microtubule binding in response to changes in Gli

261 concentration.

\section{Synergistic localization of Kif7 and Gli2 at the distal cilia tips}

Our pull-down and in vitro experiments suggested that Kif7 can interact with both the N-

265 terminal repressor as well as the zinc finger domain of Gli2. We examined the interaction between

266 full-length Gli2 and Kif7 in the cellular context. When overexpressed individually Kif7-FL is

267 exclusively localized in the cytoplasm and Gli2-FL is largely in the nucleus with a small fraction

268 localized in cytoplasmic puncta and not on microtubules. Co-transfection of Kif7-FL-mRuby with

269 mNeonGreen-Gli2-FL in HeLa cells revealed localization of both Kif7 and Gli2 along filamentous

270 microtubules in the cytosol (Fig. 5a).

271 Kif7 and Gli2 are known to accumulate at the cilium tip in response to Hedgehog (Hh)

272 pathway activation, and Kif7 is thought to form a stable platform essential for proper recruitment

273 of Gli to the cilium tip ${ }^{25,30}$. Contrary to this model, our biochemical analysis of the Kif7-Gli

274 interaction on microtubules suggest that the cilium localization of Kif7 may in turn be Gli-

275 dependent. To examine this, we measured the amount of Kif7 at the cilium tip in Gli2-/- and Gli2-

276 /-Gli3-/- cell lines (immortalized MEFs) compared to wild-type (WT) MEFs, upon stimulation

277 with the Smoothened agonist SAG (Hh pathway agonist). We found that the amount of Kif7 at 
278 cilia tips during Hh pathway activation depends on Gli. Gli2-/- cells showed 4 -fold less Kif7 in

279 the cilia, whereas Gli2-/-Gli3-/- cells showed 5-fold less Kif7 at the cilia tips compared to WT

280 cells (Fig. 5b,c). Thus, by modulating the level of Kif7 at the cilium tip, Gli acts as a master

281 regulator of its own recruitment to the cilia tip for Hh pathway activation.

282 Together these findings suggest that the Kif7-Gli2 interaction is not only sufficient to

283 recruit Gli onto microtubules, but it also positively regulates the microtubule-binding of Kif7 by

284 increasing the amount of motor on microtubules and at the cilium tip. This positive regulation in

285 turn increases the amount of Gli2 recruited to microtubules. Consequently, the interaction between

286 Gli2 and Kif7 concentrates both proteins to higher levels on microtubules in a Gli-concentration

287 dependent manner.

Re-engineering the DNA-mimicking coiled-coil domain of Kif7 as a tool for sequestration of

290 Gli away from the nucleus

Small coiled-coil domains and helical peptides have the potential to be developed as

292 inhibitors or cellular sequestration tools to tune the levels of a transcription factor in the nucleus.

293 Given the high affinity of the DNA mimicking Kif7-CC domain for Gli2, we wondered if this

294 domain could be retooled to sequester Gli away from the nucleus ${ }^{41-43}$. To test this possibility, we

295 engineered the Kif7-CC to contain an endoplasmic reticulum (ER) retention tag and mRuby at the

296 C-terminus and expressed it along with full-length mNeonGreen-Gli2 from a single mRNA in a

297 dual expression vector. The identical vector expressing mNeonGreen-Gli2 alone served as a

298 control. These constructs were transfected in HeLa cells and the amount of Gli2 in the nucleus was

299 measured as a percentage of total Gli expression levels using the mNeonGreen fluorescence

300 intensity. The localization of mNeonGreen-Gli2 in control experiments was largely nuclear 
$301(>75 \%)$ whereas, in the presence of ER-localized Kif7-CC-mRuby the nuclear mNeonGreen-Gli2

302 decreased to $<25 \%$ (Fig. 6a,b). Colocalization of fluorescent signals for Gli2 and Kif7-CC showed

303 that ER-linked Kif7-CC-mRuby sequesters Gli2 in the cytoplasm. We also found that the

304 sequestration of Gli2 in the cytoplasm (and thus away from the nucleus) depends on the level of

305 Kif7-CC-mRuby expression in these cells. Cells with higher Kif7-CC expression showed lower

306 levels of nuclear Gli2 and hence higher levels of cytoplasmic Gli2 sequestration (Fig. 6c).

307 To test if the ER-tagged Kif7-CC could inhibit the cilium tip localization of Gli2 in

308 response to Hedgehog pathway activation we transfected this construct into NIH3T3 cells. The

309 cilium tip localization of Gli2 in Kif7-CC-ER transfected cells, induced with the Hedgehog

310 pathway agonist SAG, was measured using a directly labeled Gli2 antibody that was further

311 cleaned using Gli2-/- MEFs. Our results show that the Kif7-CC-ER inhibits the levels of Gli2 at

312 the cilia tips during pathway activation compared to non-transfected wild type cells (Fig. 6d,e).

313 These findings show that the Kif7-CC peptide can be re-engineered for sequestration of 314 endogenous and over-expressed Gli in the cytoplasm and inhibit its nuclear and cilium localization. 


\section{Discussion}

Our study reports the discovery of DNA-mimicry as a mechanism for regulating nuclear

317 proteins in the cytoplasm of eukaryotes and provides critical insights into mechanisms that link

318 the Hh signaling pathway and the microtubule cytoskeleton.

319 Coiled-coils are among the most ubiquitous structural folds in proteins ${ }^{44}$. The Kif7-CC is

320 characterized by a highly negatively charged rod-like structure that competes for the same binding

321 sites on Gli2-ZF as DNA. Thus, the properties of Kif7-CC are consistent with that of a DNA-

322 mimicking protein ${ }^{45-48}$. The zinc-finger domain of Gli2 binds DNA in the nucleus and is

323 repurposed for Kif7-mediated cytoplasmic localization through the same structural design

324 principle (Fig. 7a). DNA mimicry by the Kif7 coiled-coil is the first example of a coiled-coil

325 domain in an endogenous eukaryotic protein used to bind the DNA-binding domain of a

326 transcription factor and regulate its function in the cytoplasm. A handful of examples of DNA

327 mimicking proteins described in eukaryotes are confined to the nucleus ${ }^{49,50}$ or function during

328 mitosis ${ }^{51}$. Additionally, unlike reported DNA mimics that resemble bent/distorted/single stranded

329 DNA, Kif7-CC mimics dsDNA ${ }^{49,50,52-54}$. Our observations raise the intriguing possibility that

330 cytoskeletal proteins can be integral in regulating other nuclear proteins, especially transcription

331 factors, in the cytoplasm, by mimicking double stranded DNA.

We find that Gli binds to two unusual cargo-binding sites on Kif7 - the coiled coil

333 dimerization domain and the motor domain, and the Kif7-Gli interaction regulates the Kif7-

334 microtubule affinity in a Gli dose-dependent manner (Fig. 7b). Experiments with chimeras and

335 mutant that specifically perturb Gli binding to either the coiled-coil or the motor domain in a Kif7-

336 DM provide mechanistic insights into how Gli may regulate the Kif7-microtubule interaction. Our

337 results suggest that in the absence of Gli, the coiled-coil domain of Kif7 may inhibit the Kif7- 
microtubule interaction. Gli binding to the coiled-coil interaction site on Kif7 alleviates this inhibition. The maximum dynamic range of the Kif7-microtubule binding response to Gli is only observed in the context of the wild type Kif7-DM as replacing the motor domain of Kif7 with that of Kif 27 reduces the response. Thus, of the two sites on Kif7 that play a role in recruiting Gli, it is unexpectedly the Gli binding site further away from the motor domain that has a dominant role in

343 Kif7-microtubule binding response. In general, cargo binding to the coiled-coil or the motor

344 domain of kinesins is rare. Particularly, cargo-motor domain interactions typically have an inhibitory effect on the kinesin-microtubule binding and motility ${ }^{55-58}$, which is different from our

346 observations with the Kif7-Gli interaction. It is noteworthy, that the Drosophila homolog of Kif7,

347 Costal2 is also proposed to have multiple binding sites for $\mathrm{Ci}^{59,60}$. It is possible that the multi-site

348 interaction may be beneficial for concentrating low copy number transcription factors of the Hh 349 pathway, on microtubules.

351 levels of Kif7 at the cilium tip, Gli acts as a master regulator of its own recruitment to the cilia tip

352 for its activation. Like other transcription factors, Gli is a low copy number and tightly regulated

353 protein, hence the synergistic feedback between Kif7-Gli and Kif7-microtubule interactions is a

354 mechanism that would be advantageous in rapidly concentrating Gli, to the cilium tip upon Hh

355 activation. A similar strategy may be used to concentrate and promote the formation of Gli

356 repressor at the base of the cilium when the Hh pathway is off. Interestingly, even in Drosophila,

357 where the Hh pathway is cilium-independent, the Kif7 homolog Costal2 is proposed to tether the

358 Gli homolog $\mathrm{Ci}$ to the cytoplasmic microtubules during pathway repression ${ }^{5}$. From an evolutionary

359 perspective, our results indicate that despite the divergence in the utilization of cilium between

360 vertebrates and invertebrates, the fundamental principles by which the microtubule cytoskeleton 
361 has been co-opted to regulate Hedgehog signal transduction remains conserved ${ }^{60}$. Our findings

362 also validate that Kif7 and not Kif27 is the mammalian ortholog of fly Costal2 protein that tethers

363 Gli to microtubules.

364 Gli is the final downstream effector molecule of the Hh signaling pathway and it is

365 therefore a target for regulation of the pathway activity. Aberrant activation of Gli has been

366 reported in multiple human cancers including basal cell carcinomas and glioblastomas through

367 both Hh-dependent and independent mechanisms ${ }^{12,61,62}$. An inhibitor that directly targets Gli has

368 therapeutic potential in various cancers with Gli overexpression irrespective of their etiology ${ }^{63}$.

369 Our biochemical analyses of Kif7-Gli interaction and proof-of-principle sequestration experiments

370 suggest that a small coiled-coil peptide from Kif7 can be exploited to sequester Gli in the

371 cytoplasm and restrict its entry into the nucleus (Fig. 7c). This strategy could be further developed

372 to downregulate Gli activity as well as a tool for cell biological and optogenetic studies ${ }^{41-43}$. The

373 structural analyses of the Kif7-Gli interaction opens the possibility of a new site on Gli that can be

374 exploited for drug design. On a wider scale, it represents a possible strategy for inhibiting other

375 zinc-finger proteins that play critical roles in gene regulation and may be erroneously activated in

376 disease. 


\section{Methods}

\section{$378 \quad$ Pull-down assay}

379 Plasmids for the pull-down assay were constructed by cloning human Kif7 and Gli2 sequences 380 into pCMV-BICEPTM-4 expression vector followed by truncations using InFusion HD Cloning Kit 381 (Takara). Kif7 fragments were cloned at the MCS1 to express FLAG-tagged Kif7 protein and Gli2 382 fragments were cloned at the MCS2 to express c-myc-tagged Gli2 protein. Point mutations were 383 made using the Q5® Site-Directed Mutagenesis Kit (NEB). Expi293 $\mathrm{F}^{\mathrm{TM}}$ cells were maintained in 384 Expi293 Expression medium and cultured under $7 \% \mathrm{CO}_{2}, 93 \%$ air condition at $37^{\circ} \mathrm{C}$ with $220 \mathrm{rpm}$ 385 shaking. Expi293 cells were transfected with plasmids using ExpiFectamine ${ }^{\mathrm{TM}} 293$ Reagent, 386 grown for 18-22 hours followed by addition of ExpiFectamine ${ }^{\mathrm{TM}} 293$ Transfection Enhancer 1 and 387 grown for another 24 hours. Next the cells were harvested by centrifugation at $2500 x \mathrm{~g}$ for $5 \mathrm{~min}$, 388 washed with phosphate buffered saline (PBS) and resuspended in lysis buffer (25mM Tris- $\mathrm{HCl}$, $75 \mathrm{mM} \mathrm{NaCl}, 1 \mathrm{mM} \mathrm{MgCl} 2,0.5 \mathrm{mM}$ EDTA, $100 \mu \mathrm{M}$ ATP, $1 \mathrm{mM}$ DTT, $0.1 \%$ TritonX100 and Halt $^{\mathrm{TM}}$

390 protease inhibitor cocktail; $\mathrm{pH}=7.5$ ). Cells were lysed by a short sonication and the lysate was 391 cleared by centrifugation at $125000 x g$ for 30 mins. Anti-FLAG M2 magnetic beads (Sigma

392 Aldrich) pre-equilibrated in the lysis buffer was incubated with the cleared cell lysate (labeled 393 'input') for $1 \mathrm{hr}$ at $4^{\circ} \mathrm{C}$. DynaMag-Spin was used to collect the supernatant labeled 'flow through' 394 and the beads were washed with the buffer 5 times. The last supernatant from the washes was used 395 as the 'wash' sample. The final 'bead' sample was prepared by resuspending the beads in $\sim 50 \mathrm{ul}$ 396 of buffer. The input, flow through, wash and bead samples were run on SDS-polyacrylamide gel 397 and subjected to Western blotting. Binding was detected using anti-c-myc antibody (Sigma 398 Aldrich) and the expression of the bait-protein was detected from the input sample with anti-FLAG 399 antibody (Sigma Aldrich). 


\section{Protein Expression and Purification}

402 The N-terminal fragment of Kif7 (Uniprot Q2M1P5) (Kif7-DM 1-543aa) was cloned into a 403 pFastBac expression vector (Thermo) that included a tobacco etch virus (TEV) protease cleavable 404 N-terminal 6x His-tag and SUMOstar solubilization tag. To determine which residues to swap in 405 the chimeras, we first performed homology modeling of the two kinesins using PROMALS 3D 406 (UTSW). We complimented this with modeling of Kif7 and Kif27 CC domains using COILS 407 (EMBL) and PairCoil (MIT) to make the most accurate coiled-coil substitution possible. For the 408 Kif7M-Kif27-CC chimera, Kif7 1-462aa was used for the motor and Kif27 475-570aa was used 409 for the coiled-coil. For the Kif27M-Kif7-CC chimera, Kif27 1-475aa was used for the motor and 410 Kif7 460-600aa was used for the coiled-coil. Dimeric Kif7 N-terminal constructs: Kif7-DM (1411 543aa), Kif7-DME502A and the Kif7-Kif27 chimeric constructs were expressed in SF9 insect cell

412 line using the Bac-to-Bac ${ }^{\circledR}$ Baculovirus Expressions System (Thermo) with cells grown in 413 HyClone CCM3 SFM (GE Life Sciences) and expressed from P3 virus for $72 \mathrm{H}$ at $27^{\circ} \mathrm{C}$. 414 Monomeric Kif7 constructs (Kif7-MM 1-398aa) containing the motor domain was cloned into a 415 modified pET-21-a expression vector that contained a TEV protease cleavable N terminal 6x His416 tag. Kif7 monomers were expressed in BL21 (DE3) Rosetta (Millipore) E. coli at $18^{\circ} \mathrm{C}$ with $417 \quad 0.25 \mathrm{mM}$ IPTG for 18-20H. Dimeric and monomeric kinesin pellets containing the motor domain 418 of Kif7 were lysed by short sonication in buffer A (50mM phosphate pH $8.0300 \mathrm{mM} \mathrm{NaCl} 5 \%$ 419 glycerol, $1 \mathrm{mM} \mathrm{MgCl}_{2}$ and $25 \mathrm{mM}$ imidazole) supplemented with $0.15 \%$ tween, $0.5 \%$ Igepal, $420100 \mu \mathrm{M}$ ATP 2mM TCEP, 1mM PMSF, $75 \mathrm{U}$ benzonase and 1X HALT (Thermo). Lysate was 421 cleared by ultracentrifugation and supernatant was incubated with Ni-NTA for 1H. Resin was 422 washed with buffer A supplemented with $20 \mu \mathrm{M}$ ATP and $0.5 \mathrm{mM}$ TCEP and eluted with $400 \mathrm{mM}$ 
423 imidazole with $100 \mu \mathrm{M}$ ATP. Peak fractions were pooled and, if needed, cleaved overnight by TEV

$424(1 / 30 \mathrm{w} / \mathrm{w})$ at $4^{\circ} \mathrm{C}$. Proteins were further purified by size exclusion chromatography (Superdex 200

425 10/300GL) in 50mM HEPES pH $7.4300 \mathrm{mM} \mathrm{NaCl} \mathrm{5 \%} \mathrm{glycerol} \mathrm{5mM} \mathrm{ß-Me} 1 \mathrm{mM} \mathrm{MgCl}_{2}$ and

$426100 \mu \mathrm{M}$ ATP and frozen in liquid nitrogen. Dimerization of a given construct was determinable by

427 gel filtration profiles.

428 The coiled-coil dimerization domain fragment of Kif7, (Kif7-CC 460-600aa) was cloned into a 429 modified pGEX vector that contained a human rhinovirus (HRV) protease cleavable $\mathrm{N}$ terminal

430 GST-tag. The point mutants of Kif7-CC were generated by site-directed mutagenesis using In-

431 Fusion HD cloning kit (Takara). The Kif7 SCC construct (aa488-540) was ordered as a gene-

432 fragment (Genscript) and cloned into pETDuet-1 by In-Fusion HD Cloning Kit. All Kif7-CCs and

433 Kif7-SCC were expressed in BL21 (DE3) Rosetta (Millipore) E. coli at $18^{\circ} \mathrm{C}$ with $0.25 \mathrm{mM}$ IPTG

434 for 18-20hr. The cell pellets were lysed by short sonication in buffer B (PBS pH 7.3, 5\% glycerol)

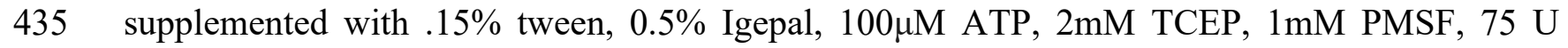

436 benzonase and 1X HALT (Thermo). Lysate was cleared by ultracentrifugation and supernatant

437 was incubated with GST-4B beads (GE Life Sciences) for 1H. Resin was washed with buffer B

438 supplemented with $0.5 \mathrm{mM}$ TCEP and eluted with $10 \mathrm{mM}$ reduced glutathione in $40 \mathrm{mM}$ PIPES pH

$4397.3150 \mathrm{mM} \mathrm{NaCl}$. Peak fractions were pooled and, if needed, cleaved overnight by GST-HRV-3C

$440 \quad(1 / 40 \mathrm{w} / \mathrm{w})$ at $4^{\circ} \mathrm{C}$. Proteins were further purified by size exclusion chromatography (Superdex 200

441 10/300GL) in 40mM PIPES pH $7.3150 \mathrm{mM} \mathrm{NaCl} \mathrm{5 \%} \mathrm{glycerol} \mathrm{2mM} \mathrm{TCEP} \mathrm{and} \mathrm{frozen} \mathrm{in} \mathrm{liquid}$

442 nitrogen.

443 The zinc-finger domain of Gli2 (Uniprot P10070) (Gli2-ZF 418-604aa) was cloned into a modified

444 pET-Duet-1 expression vector that contained an HRV protease cleavable N terminal 6x His-tag.

445 Truncations were made using the InFusion HD Cloning Kit (Takara). The same construct was 
446 cloned into a modified pET-Duet-1 vector that contained an HRV protease cleavable $\mathrm{N}$ terminal

447 6x His-tag, and a C-terminal SNAP/GFP tag. Gli2-ZF protein was expressed in BL21 (DE3)

448 Rosetta (Millipore) E. coli at $18^{\circ} \mathrm{C}$ with $0.25 \mathrm{mM}$ IPTG for $18-20 \mathrm{hr}$. Gli2-ZF pellets were lysed by

449 short sonication in buffer C (40mM PIPES pH $7.3150 \mathrm{mM} \mathrm{NaCl})$ supplemented with .15\% tween,

$450 \quad 0.5 \%$ Igepal, 2mM TCEP, 1mM PMSF, $300 \mathrm{U}$ benzonase and 1X HALT (Thermo). Lysate was

451 cleared by ultracentrifugation and supernatant was incubated with Ni-NTA for 1H. Resin was

452 washed with buffer A supplemented with 0.5mM TCEP and eluted with 400mM imidazole. Peak

453 fractions were pooled and, if needed, cleaved overnight by GST-HRV-3C $(1 / 40 \mathrm{w} / \mathrm{w})$ at $4^{\circ} \mathrm{C}$.

454 Proteins were further purified by ion-exchange chromatography using a Heparin column; Gli2-ZF

455 proteins are DNA binding and generally elute from the heparin column around $1 \mathrm{M} \mathrm{NaCl}$. Proteins

456 were further purified by size exclusion chromatography (Superdex 75 10/300GL and Superdex

457200 10/300GL) in 40mM PIPES pH 7.3 150mM NaCl 5\% glycerol 2mM TCEP and frozen in

458 liquid nitrogen.

459 All proteins were greater than 95\% pure and eluted as a single peak from the size exclusion

460 chromatography column.

Bio-Layer Interferometry Assays

463 BLI experiments were performed in an Octet Red 96 instrument (ForteBio). To quantify Kif7-Gli2

464 binding, Experiments were performed in an assay buffer containing 40mM TRIS pH7.3, 40mM

$465 \mathrm{KCl}, 40 \mu \mathrm{M}$ ATP $1 \mathrm{mM} \mathrm{MgCl} 2,0.12 \%$ tween and $0.5 \mathrm{mM}$ TCEP. In the simplest assay, each dimeric

466 GST-Kif7-CC protein was immobilized on an anti-GST biosensor chip at a concentration of

467 120nM. Unbound Kif7 was washed away with buffer, and the chips were then dipped into buffer

468 with a range of Gli2-ZF concentrations from $0-1 \mu \mathrm{M}$ to allow for association of the two proteins. 
After 300 seconds, they were then moved back into a well with just buffer to allow dissociation

470 for 400 seconds. The equilibrium response of each sensor from three independent repeats at each

471 Gli2 concentration were normalized and plotted against Gli2 concentration and fitted to the

472 following Hill function: $Y=\left(B_{\max }{ }^{*} X^{h}\right) /\left(K_{d}{ }^{h}+X^{h}\right)$; where $B_{\max }$ is the maximum specific binding, $K_{d}$

473 is the ligand concentration needed to achieve a half-maximum binding at equilibrium and $\mathrm{h}$ is the

474 Hill slope.

475 The DNA competition experiments were performed in the same way with one notable exception:

$476125 \mu \mathrm{M}$ TRE-2S DNA (a Gli2 DNA binding target) was held constant along the Gli2 titration from

$477 \quad 0-1 \mu \mathrm{M}$. The assays were also performed with HIS-tagged Kif7-DM, Kif7-DME502A and the Kif7-

478 Kif27 chimeric proteins, requiring the use of an anti-penta-HIS biosensor chip, and referencing

479 sensors to account for non-specific binding of Gli2 to the HIS sensor. A flipped assay, using HIS-

480 tagged Gli2 on the sensor and a range of Kif7 concentrations in buffer, was also conducted.

\section{Kif7-Gli2 Complex Stoichiometry Determination}

483 The Kif7-CC and Kif7-DM protein samples were not amenable to dynamic light scattering 484 analysis. To determine the stoichiometry of the Kif7-Gli2 complexes, we purified GFP-tagged 485 constructs of Kif7-DM, Kif7-CC, and Gli2-ZF. Next, the Kif7 (Kif7-CC or Kif7-DM) and Gli2 486 were mixed at high concentration with Gli2-ZF in molar excess $(125 \mu \mathrm{M}$ Kif7 and $750 \mu \mathrm{M}$ Gli2$487 \mathrm{ZF}$ ) and incubated for 15 minutes on ice. After incubation, $200 \mu \mathrm{L}$ of this mix was injected into a 488 Superdex 200 10/300 GL column. Fractions that contained complexed protein were determined by 489 elution volume shift in the gel filtration chromatogram. Complex containing fractions were heated 490 at $50^{\circ} \mathrm{C}$ for 5 mins and run in SDS-PAGE and quantified in two ways: (1) Scanned the unstained 491 SDS-PAGE gel at $488 \mathrm{~nm}^{64}$. Since GFP is stable in SDS up to at least $0.5 \% \mathrm{w} / \mathrm{v}^{65}$ and SDS-PAGE 
uses $0.1 \%$, the GFP fluorescence can be quantitatively analyzed on a regular SDS-PAGE gel with

493 this sample preparation protocol ${ }^{64}$. The ratio of GFP fluorescence corresponding to the Kif7 and

494 Gli bands was measured using an Amersham ${ }^{\mathrm{TM}}$ Typhoon Scanner and the intensity analyses was

495 performed using ImageJ to obtain the stoichiometry. (2) Quantitative western blot analysis of the

496 complex was performed using a directly labeled 488nM anti-GFP antibody (Santa Cruz). The

497 fluorescence intensity at $488 \mathrm{~nm}$ was quantified using an Amersham ${ }^{\mathrm{TM}}$ Typhoon Scanner and 498 analyzed with ImageJ. The results from these two independent experiments were consistent with

499 the stoichiometries reported in this study.

\section{Structural modeling}

502 Primary sequences of the zinc-finger domain of human Gli2 (UniprotKB P10070) (Gli2-ZF, 437-

503 589aa), the coiled-coil domain of human Kif7 (UniprotKB Q2M1P5) (Kif7-SCC, 481-543aa), the

504 coiled-coil domain of human Kif27 (UniprotKB Q86VH2) (Kif27-CC, 475-570aa) and the N-

505 terminal motor domain of human Kif27 (UniprotKB Q86VH2) (Kif27M, 1-475aa) were retrieved

506 from the database in FASTA format. A preliminary search for homologs to serve as templates for 507 modeling was performed with BLAST $^{66}$ and HHBlits ${ }^{67}$ against the SWISS-MODEL template

508 library (SMTL). The following templates with the highest quality predictions (based on target509 template alignment) were selected for homology modeling: (i) Gli1 zinc finger (PDB: 2GLI) for

510 Gli2-ZF (ii) ROCK1 coiled-coil (PDB: 3O0Z) for Kif7-CC and Kif27-CC (iii) Kif7 motor domain

511 (PDB: 6MLQ) for Kif27M. The 3D structure of these protein domains was built using ProMod3

512 in the SWISS-MODEL server ${ }^{68,69}$. The global and per-residue model quality was assessed using

513 GMQE and QMEAN scoring function ${ }^{70}$. The structural models were visualized in UCSF

514 Chimera $^{71}$. Electrostatic potential of the surfaces were generated from the APBS server ${ }^{72}$. 
515 For obtaining the structural model of the Kif7-SCC:Gli2-ZF protein complex, the Gli2-ZF model

516 was docked on the Kif7-CC model using an automated server, ClusPro $2.0^{73}$, in which receptor

517 was Kif7-SCC and Gli2-ZF was used as a ligand. Based on different desolvation and electrostatic

518 potential, ClusPro 2.0 can differentiate thousands of conformations of the protein. The generated

519 conformations were further categorized through clustering and 8 most fit structures (which are

520 found to be closest to native structure from X-ray crystallography results) were considered. The

521 interface areas in the structural model of the protein complex were calculated by the PDBsum

522 webserver ${ }^{74}$. These contact residues were mutated to Ala for binding analysis to assess the

523 predicted models.

525 Cryo-EM Sample Preparation

526 For grid preparation, all Kif7-Gli2 preparations were diluted using BRB80 (80 mM 1,4-

527 piperazinediethanesulfonic acid [PIPES], $\mathrm{pH} 6.8,1 \mathrm{mM} \mathrm{MgCl}_{2}, 1 \mathrm{mM}$ ethylene glycol tetraacetic 528 acid [EGTA]). Porcine brain microtubules were prepared from frozen aliquots of $5 \mathrm{mg} / \mathrm{ml}$ tubulin

529 (Cytoskeleton, Denver, CO) in polymerization buffer (BRB80; $80 \mathrm{mM}$ PIPES, pH 6.8, $1 \mathrm{mM}$

530 EGTA, $2 \mathrm{mM} \mathrm{MgCl}$, 3mM GMP-CPP, 10\% dimethyl sulfoxide (DMSO)) at $37^{\circ} \mathrm{C}$ for $2-3$ hours.

531 The polymerized microtubules were then incubated at room temperature for several hours or 532 overnight before use. The following day or several hours later the microtubules were spun down 533 on a bench top centrifuge 14,00 RPM 10min to pellet. The pellet was resuspended in 534 polymerization buffer without DMSO. The Kif7 dimer and monomer preparations were made by 535 mixing the Kif7 with the Gli2 or Gli2-SNAP for 1 hour prior to incubation with the microtubules. 536 Kif7-dimer $(0.36 \mathrm{mg} / \mathrm{ml})$ plus Gli2 $(0.2 \mathrm{mg} / \mathrm{ml})$ in BRB plus $2 \mathrm{mM} \mathrm{ADP,} 2 \mathrm{mM} \mathrm{MgCl}_{2}$, $\mathrm{Kif7}_{-}$ 

AMPPNP and $1 \mathrm{mM} \mathrm{MgCl}$.

540 Grids were glow-discharged before sample application. The cryo-samples were prepared using a

541 manual plunger, which was placed in a homemade humidity chamber that varied between 80 and

$54290 \%$ relative humidity. A $4-\mu 1$ amount of the microtubules at $\sim 0.5 \mu \mathrm{M}$ in BRB80 was allowed to

543 absorb for $1 \mathrm{~min}$, and then $4 \mu \mathrm{l}$ of the different Kif7-dimer or monomer plus Gli2 with and without

544 SNAP were incubated with microtubules on the grid. After a short incubation of 2 min, the sample

545 was blotted (from the back side of the grid) and plunged into liquid ethane.

\section{$547 \quad$ EM image acquisition and data processing}

548 Images of frozen-hydrated Kif7-microtubule complexes (see Extended Data Table 1) were 549 collected on a Titan Krios (FEI, Hillsboro, OR) operating at $300 \mathrm{keV}$ or an Arctica (FEI, Hillsboro,

550 OR) equipped with a K2 Summit direct electron detector (Gatan, Pleasanton, CA). The data were

551 acquired using the Leginon automated data acquisition ${ }^{75,76}$. Image processing was performed

552 within the Appion processing environment ${ }^{76,77}$. Movies were collected at a nominal magnification

553 of $29000 \times$ and $36000 x$ with a physical pixel size of 1.03 and $1.15 \AA$ /pixel respectively. Movies

554 were acquired using a dose rate of $\sim 4.2$ and 5.6 electrons/pixel/second over 9 and 8 seconds

555 yielding a cumulative dose of $\sim 36$ and $\sim 34$ electrons $/ \AA^{2}$ (respectively). The MotionCor frame

556 alignment $\operatorname{program}^{78,79}$ was used to motion-correct. Aligned images were used for CTF

557 determination using CTFFIND $4^{80}$ and only micrographs yielding CC estimates better than 0.5 at

$5584 \AA$ resolution were kept. Microtubule segments were manually selected, and overlapping

559 segments were extracted with a spacing of $80 \AA$ along the filament. Binned boxed segments 
$560(2.05 \AA /$ pixel, 240-pixel box size for the Krios data and $2.30 \AA /$ pixel, 192 pixel box size for the 561 Arctica data) were then subjected to reference-free 2D classification using multivariate statistical

562 analysis (MSA) and multi-reference alignment (MRA) ${ }^{79,81}$. Particles in classes that did not clearly

563 show an $80 \AA$ layer line were excluded from further processing.

\section{Cryo-EM $3 D$ reconstruction}

566 Undecorated 13,14- and 15-protofilament microtubule densities ${ }^{82}$ were used as initial models for

567 all preliminary reconstructions. We used the IHRSR procedure ${ }^{83}$ for multimodel projection 568 matching of microtubule specimens with various numbers of protofilaments ${ }^{84}$, using libraries from

569 the EMAN2 image processing package ${ }^{85}$. After each round of projection matching, an asymmetric

570 backprojection is generated of aligned segments, and the helical parameters (rise and twist)

571 describing the monomeric tubulin lattice are calculated. These helical parameters are used to

572 generate and average 13, 14 and 15 symmetry-related copies of the asymmetric reconstruction, 573 and the resulting models were used for projection matching during the next round of refinement.

574 The number of particles falling into the different helical families varied. Helical families that had 575 enough segments were further refined. Final refinement of microtubule segment alignment 576 parameters was performed in FREALIGN ${ }^{86}$ without further refinement of helical parameters. The 577 data is unbinned during refinement. The boxsize of the Krios data was binned from 480 to 380 578 reducing the pixel size from 1.03 to $1.3 \AA$ pixel. FSC curves were used to estimate the resolution 579 of each reconstruction, using a cutoff of 0.143 . To better display the high-resolution features of

580 the 3D map shown, we applied a B-factor of $150 \AA$, using the program bfactor 581 (http://grigoriefflab.janelia.org). 


\section{Model building in the EM map}

584 The EM-derived structure of AMPPNP-Kif7 bound to microtubules (PDB: 6 MLR, EMD: 9141)

585 was fit as a rigid body into the density for AMPPNP-Kif7 dimer bound to microtubules in the 586 presence of Gli2-SNAP, using the 'Fit in Map' utility in UCSF Chimera ${ }^{71}$. To locate the density

587 corresponding to the Gli2 protein, cryo-EM maps for ADP-Kif7 dimer with Gli2, AMPPNP-Kif7

588 monomer with Gli2-SNAP and AMPPNP-Kif7 dimer with Gli2-SNAP were superposed via the

589 Kif7 motor domains, and the minimal density common to all three maps was identified. To

590 construct a structural model for Gli2 to fit the density, individual zinc fingers were first extracted

591 from the crystal structure of a five-finger Gli1 in complex with DNA (PDB:2GLI). Next, various

592 combinations of zinc fingers were fit into the minimal density corresponding to Gli2 and the best

593 model was identified through its model-map correlation coefficient and visual inspection. Since

594 the density corresponding to each zinc finger could be fit by any one of the five zinc-fingers of

595 Gli2, the partial structure for Gli2 was modeled as a Poly-Ala sequence. Finally, flexible fitting

596 for the structure comprising of Gli2 zinc fingers, AMPPNP-bound Kif7 motor and $\alpha-\beta$ tubulin

597 heterodimer was performed with phenix.real_space_refine ${ }^{87}$. The final structure has a model-map

598 correlation of 0.84 , bond length RMSD of $0.008 \AA$ and bond angle RMSD of $0.98^{\circ}$. There are no

599 outliers in the Ramachandran map. Cryo-EM reconstructions and their associated models were

600 superposed using the UCSF Chimera "Fit in map" tool".

601

\section{Total Internal Reflection Fluorescence Microscopy Assays}

603 In vitro TIRF-based microscopy experiments were carried out as described in Subramanian et al.,

$6042013^{88}$. Microscope chambers were constructed using a 24 x $60 \mathrm{~mm}$ PEG-Biotin coated glass slide

605 and $18 \times 18 \mathrm{~mm}$ PEG coated glass slide separated by double-sided tape to create two channels for 

$\mathrm{MgCl}_{2}$ and 1mM EGTA), 1mMATP, 1mMGTP, 0.1\%methylcellulose and 3\%sucrose. For all experiments, oxygen scavenging mix (OS) comprising of $25 \mathrm{mM}$ glucose, $40 \mathrm{mg} / \mathrm{ml}$ glucose oxidase, $35 \mathrm{mg} / \mathrm{ml}$ catalase and $0.5 \%$ beta-mercaptoethanol was included in the final buffer. Images were acquired using NIS-Elements (Nikon) and analyzed using ImageJ.

611 Microtubule binding assay: X-rhodamine/HiLyte 647 (1:10 labeled to unlabeled) and biotin (1:10

612 labeled to unlabeled) labeled microtubules were polymerized in the presence of GMPCPP, a non613 hydrolysable GTP-analogue, and immobilized on a neutravidin coated glass coverslip. Coverslips

614 were briefly incubated with casein to block non-specific surface binding before addition of 100nM 615 kinesin and/or varying concentrations of Gli2-ZF in assay buffer and antifade reagent 616 (25mMglucose, $40 \mathrm{mg} / \mathrm{ml}$ glucose oxidase, $35 \mathrm{mg} / \mathrm{ml}$ catalase and $0.5 \% \beta$-mercaptoethanol). 617 Images were acquired from multiple fields of the same chamber.

618 Single molecule assays: Initial fluorescence intensity analysis to determine the oligomerization 619 state of the GFP tagged proteins was performed by immobilizing GFP-tagged protein non620 specifically to a glass coverslip. After washing away unbound protein, BRB80-DTT buffer 621 supplemented with OS mix was added to the chamber and images were acquired at $0.37 \mathrm{~s}$ intervals 622 on an ANDOR iXon Ultra EMCCD camera with EM gain set to 287. Fluorescent spots were 623 selected by visual inspection and the average intensity of the first five frames was used to compute 624 the initial intensity after background subtraction.

625 Interaction of single Kif7-DM-GFP molecules with microtubules in the presence of Gli2-ZF was 626 analyzed by immobilizing biotinylated microtubules to a glass coverslip as described earlier, 627 followed by addition of Kif7-DM-GFP $(1 \mathrm{nM}$ or $0.1 \mathrm{nM})$ with increasing concentrations of Gli2$628 \mathrm{ZF}(0 \mathrm{nM}, 25 \mathrm{nM}, 50 \mathrm{nM})$ and images were acquired at $0.37 \mathrm{~s}$ intervals on an ANDOR iXon Ultra 
EMCCD camera with EM gain set to 287. The density of tracks in the $1 \mathrm{nM}$ Kif7-DM-GFP data

630 set were too high to perform tracking analysis, so we repeated the experiment with $0.1 \mathrm{nM}$ Kif7-

631 DM-GFP and used that data for analyses.

\section{Co-sedimentation of Kif7 on GDP-Taxol Stabilized Microtubules}

634 GDP-taxol stabilized microtubules were prepared from purified pre-cleared bovine tubulin as 635 described in Subramanian et al., $2010^{89}$. Kif7-DM-GFP at $1 \mu$ M concentration were incubated with 636 microtubules $(0-10 \mu \mathrm{M})$ in the presence of $1 \mathrm{mM} \mathrm{ATP}, 2 \mathrm{mM} \mathrm{MgCl}$, with or without $5 \mu \mathrm{M}$ Gli

637 for 15 minutes at room temperature in 1x BRB80 supplemented with $0.1 \mathrm{mg} / \mathrm{mL}$ BSA and then 638 subjected to sedimentation by ultracentrifugation at $50,000 \mathrm{rpm}$ for 10 minutes at $27^{\circ} \mathrm{C}$ in a TLA 639120.1 rotor (Beckman Coulter). Pellets were subsequently resuspended and the amount of protein 640 in each pellet and supernatant was analyzed by SDS-PAGE. Proteins were stained using Gel 641 Code $^{\mathrm{TM}}$ Blue Stain (Thermo Fischer Scientific) and scanned using GE Typhoon FLA 9000 Gel 642 Scanner. Dissociation constants were calculated by fitting of the experimental data to a Hill 643 equation using GraphPad Prism.

646 The coiled-coil dimerization domain fragment of human Kif7, (Kif7-CC 460-600aa) expressed 647 and purified for the biochemistry experiments was used to produce antibodies in rabbits from 648 Covance Inc. (Princeton, NJ). The Kif7 antiserum was affinity-purified using antigen conjugated 649 CNBr-Sepharose resins. The cDNA sequence encoding 1053-1264 aa mouse was amplified and 650 cloned upstream of sequences encoding a polyhistidine stretch (His) tag in pET23b (Novagen). 651 The mGli2-His recombinant protein was expressed in BL21 (DE3) pLysS cells (Stratagene) and 
652 purified under denaturing condition ( $8 \mathrm{M}$ urea) using the TALON metal affinity resin from

653 Clontech. Purified fusion proteins were used to produce antibodies in guinea pigs from Covance

654 Inc. (Princeton, NJ). The Gli2 antiserum was affinity-purified using antigen conjugated CNBr-

655 Sepharose resins. The Kif7 and Gli2 antibodies were further 'cleaned' by incubation with fixed

$656 \mathrm{Kif7}-/-$ and Gli2-/- MEFs respectively to reduce non-specific staining in immunofluorescence

657 experiments and labeled with Alex Fluor 647 dye using Molecular Probes kit.

\section{Cell culture, transfections, and immunofluorescence}

660 HeLa cells were obtained from Robert Kingston (Massachusetts General Hospital). COS7 and

661 NIH3T3 cells were purchased from ATCC and BPS Biosciences respectively. MEFs (WT, Gli2-

662 /-, Gli2-/-Gli3-/-, Kif7-/-) were obtained from Kathryn Anderson (Sloan Kettering Institute),

663 Robert Lipinski (University of Wisconsin), Adrian Salic (Harvard Medical School), and Stephane

664 Angers (University of Toronto). HeLa, NIH3T3 and MEF cells were maintained in high-glucose

665 Dulbecco's modified Eagle's medium (DMEM) supplemented with 10\% fetal bovine serum

666 (FBS), sodium pyruvate $(1 \mathrm{mM})$ and L-glutamine $(2 \mathrm{mM})$. Cells were cultured on clean coverslips

667 under the following conditions: $5 \% \mathrm{CO}_{2}, 95 \%$ air condition at $37^{\circ} \mathrm{C}$.

668 HeLa and COS7 cells were transfected with plasmids using jetPrime transfection reagent and

669 incubated for 18-22 hours. Next the cells were fixed using a mixture of methanol and acetone (1:1

670 in volume) for 10 minutes in $-20 \mathrm{C}$, washed with washing buffer (PBS $+0.05 \%$ Tween 20 ) and

671 mounted on 25mmX75mm glass slides with ProLong Diamond Antifade Mountant with DAPI

672 (Thermo Fisher). Z-stacks were acquired on an inverted Nikon confocal microscope using laser

673 illumination source (405nm, 488nm and 561nm channels corresponding to DAPI, Gli2-Neongreen

674 and Kif7 respectively) with a pinhole of 0.5 . 
675 For experiments with NIH3T3, cells were grown to $\sim 70$ confluency followed by transfection with

676 plasmids using jetPrime transfection reagent and incubated for 18-22 hours. For experiments with

677 MEFs, cells were grown to complete confluency. Then both cell types were serum-starved with

$678 \quad 0.2 \%$ FBS in DMEM to induce ciliogenesis for 24 hours, followed by treatment with 500nM SAG

679 for 12-18 hours before being fixed for immunofluorescence. Cells were fixed using a mixture of

680 methanol and acetone (1:1 in volume) for 10 minutes in $-20 \mathrm{C}$, washed with washing buffer

681 (PBS $+0.05 \%$ Tween 20) for 3 times, blocked with blocking buffer (PBS + 2\%BSA; OmniPur

682 BSA; EMD Millipore) for one hour at room temperature. Samples were probed overnight at 4C

683 with the following specific primary antibodies (diluted in blocking buffer): Alexa Fluor 647nm

684 labeled Kif7 antibody (1:10) (in-house), Alexa Fluor 647nm labeled Gli2 antibody (1:10) (in-

685 house), Cyanine3 or Alexa-488 labeled polyclonal $\gamma$-tubulin antibody (1:1000) (Thermo Fisher)

686 and Alexa Fluo 488nm labeled acetylated $\alpha$-tubulin antibody (1:500) (Santa Cruz Biotechnology).

687 Samples were mounted on a $25 \mathrm{mmX} 75 \mathrm{~mm}$ glass with ProLong Diamond Antifade Mountant

688 (Thermo Fisher). Z-stacks were acquired on an inverted Nikon confocal microscope using laser

689 illumination sourc.3e $(488 \mathrm{~nm}, 561 \mathrm{~nm}$ and $647 \mathrm{~nm}$ channels respectively) with a pinhole of 1 . Z-

690 projections were generated by sum of images from planes that included the entire cilium.

692 Quantification and Statistical Analysis

693 ImageJ was used to assess GFP/Alexa 647 fluorescence intensities on microtubules. For all

694 average intensity per pixel values recorded, a rectangular area along each microtubule was selected

695 with a width of 3 pixels. Background intensities were also subtracted locally from regions of

696 interest of the same area around the selected microtubule. Intensities were not analyzed for

697 microtubules found at the edges of the camera's field of view. 
698 For single molecule residence time analysis, fluorescent spots along each microtubule were

699

700 Gli2-ZF.

708 The cumulative frequency plot of residence time of Kif7-DM in the absence of Gli2-ZF was not

709 fit since a high fraction of the events in this condition have a very short the residence times of 1-2

710 frames. This indicates that the true lifetime is lower than the time resolution of our experiment 711 (0.37 s per frame). For the same reason, the Tau ${ }^{\text {Fast }}$ values are likely to represent the upper limit

712 for the lifetime of un-complexed Kif7-DM molecules.

713

714 All TIRFM data were analyzed using GraphPad Prism. "N'" numbers in experiments refer to the

715 unique number of microtubules or single kinesin molecules (as mentioned in the figure legends)

716 used for the dataset. Details of fit parameters can be found in the corresponding figure legends.

717 ImageJ was used to analyze intensity of protein bands for stoichiometry determination. A 718 rectangular box was drawn around the protein band of interest for intensity readout. Local 719 background was subtracted using a 3-pixel radius around the region of interest. 
721 Octet Data Analysis software was used to extract the binding response for BLI data. The responses

722 were plotted as a function of protein concentration and fit to Hill curves using GraphPad Prism.

723 The binding responses were normalized between 0 and 100 prior to curve fitting.

724 For structure fitting, atomic models were fit into the cryo-EM density using UCSF Chimera and 725 model to map correlation coefficients were calculated using the 'Fit in Map' utility.

727 For quantification of Kif7 intensity in the cilia, images of cilia were analyzed on ImageJ. The 728 Alexa647 intensity profile along the length of each cilium was measured from tip to base. The 729 acetylated $\alpha$-tubulin channel was used as a marker for cilia length and the $\gamma$-tubulin channel was 730 used as a marker for the base of the cilia. Intensity profiles of different cilia lengths were aligned 731 by their tips and averaged. The integrated intensity profiles were plotted using GraphPad prism.

732 Statistical details can be found in the results section and corresponding figure legends.

733 For cytoplasmic sequestration quantification, images of cells were analyzed on ImageJ. The

734 nucleus boundary points (including the nucleolus pattern) were identified by using automatic

735 thresholding on DAPI channel (Otsu method in Image J). The threshold value was obtained from

736 auto-thresholding the brightest frame of confocal z-stacks images. This threshold value was used

737 to create binary masks on individual frame of Z-stacks. These masks created by applying threshold

738 on the DAPI channel (called "DAPI Nucleus mask") were used to measure Gli2-neongreen

739 intensity in the nucleus. Finally, the nuclear Gli2-neongreen intensity was integrated after

740 background subtraction by rolling ball ( $\mathrm{r}=100$ pixels) in image $\mathrm{J}$. Next, to create a mask in the

741 Gli2-neongreen for the whole cell boundary an ImageJ macro was applied that automatically

742 determined the threshold value with the best-match mask in the nucleus area ("Gli2 Nucleus

743 mask") with "DAPI Nucleus mask". The best match was selected by finding the maximum value 
744 in XNOR operation between "Gli2 Nucleus mask" and "DAPI Nucleus mask". With the given

745 threshold value, a binary mask over the whole cell area was created ("Gli2 whole cell mask") and

746 again the Gli2-neongreen intensity in the whole cell was integrated after background subtraction

747 by rolling ball ( $\mathrm{r}=100$ pixels $)$.

748

749 Data and materials availability

750 The datasets generated and/or analyzed during the current study are available as supplemental

751 table. Any additional data are available from the corresponding author on reasonable request.

752

753 Code availability

754 All custom codes used for analyses of the data are available from the corresponding author on 755 reasonable request. 


\section{References}

7581 Liu, Y., Li, P., Fan, L. \& Wu, M. The nuclear transportation routes of membrane-bound transcription factors. Cell Communication and Signaling 16, 12 (2018).

7602 Dong, C., Li, Z., Alvarez Jr, R., Feng, X.-H. \& Goldschmidt-Clermont, P. J. Microtubule binding to Smads may regulate TGF $\beta$ activity. Molecular cell 5, 27-34 (2000).

7623 Ziegelbauer, J. et al. Transcription factor MIZ-1 is regulated via microtubule association. Molecular cell 8, 339-349 (2001).

7644 Batut, J., Howell, M. \& Hill, C. S. Kinesin-mediated transport of Smad2 is required for signaling in response to TGF- $\beta$ ligands. Developmental cell 12, 261-274 (2007).

7665 Robbins, D. J. et al. Hedgehog elicits signal transduction by means of a large complex containing the kinesin-related protein costal2. Cell 90, 225-234 (1997). in the Hedgehog signaling pathway. Cell 90, 235-245 (1997).

771

772 Wilson, C. W. \& Chuang, P.-T. Mechanism and evolution of cytosolic Hedgehog signal transduction. Development 137, 2079-2094 (2010).

8 Ingham, P. W., Nakano, Y. \& Seger, C. Mechanisms and functions of Hedgehog signalling across the metazoa. Nature Reviews Genetics 12, 393-406 (2011). proteins. Nature 426, 83-87 (2003).

10 Lee, J., Platt, K. A., Censullo, P. \& Ruiz i Altaba, A. Gli1 is a target of Sonic hedgehog that induces ventral neural tube development. Development 124, 2537-2552 (1997). mouse homologs of the Drosophila segment polarity gene cubitus interruptus, Gli, Gli-2, and Gli-3, in ectoderm-and mesoderm-derived tissues suggests multiple roles during postimplantation development. Developmental biology 162, 402-413 (1994). developmental biology 27, 513-537 (2011). Gli3 activities by an amino-terminal repression domain: implication of Gli2 and Gli3 as primary mediators of Shh signaling. Development 126, 3915-3924 (1999). an anterior/posterior repressor gradient in the developing vertebrate limb. Cell 100, 423434 (2000).

15 Wen, X. et al. Kinetics of hedgehog-dependent full-length Gli3 accumulation in primary cilia and subsequent degradation. Molecular and cellular biology 30, 1910-1922 (2010).

16 Matise, M. P., Epstein, D. J., Park, H. L., Platt, K. A. \& Joyner, A. L. Gli2 is required for induction of floor plate and adjacent cells, but not most ventral neurons in the mouse central nervous system. Development 125, 2759-2770 (1998).

17 Ding, Q. et al. Diminished Sonic hedgehog signaling and lack of floor plate differentiation in Gli2 mutant mice. Development 125, 2533-2543 (1998).

18 Kim, J., Kato, M. \& Beachy, P. A. Gli2 trafficking links Hedgehog-dependent activation of Smoothened in the primary cilium to transcriptional activation in the nucleus. Proceedings of the National Academy of Sciences 106, 21666-21671 (2009). 
80019 Zeng, H., Jia, J. \& Liu, A. Coordinated translocation of mammalian Gli proteins and suppressor of fused to the primary cilium. PloS one 5, e15900 (2010).

80220 Caspary, T., Larkins, C. E. \& Anderson, K. V. The graded response to Sonic Hedgehog depends on cilia architecture. Developmental cell 12, 767-778 (2007).

80421 Goetz, S. C. \& Anderson, K. V. The primary cilium: a signalling centre during vertebrate development. Nature Reviews Genetics 11, 331-344 (2010).

22 Liem, K. F., He, M., Ocbina, P. J. R. \& Anderson, K. V. Mouse Kif7/Costal2 is a ciliaassociated protein that regulates Sonic hedgehog signaling. Proceedings of the National Academy of Sciences 106, 13377-13382 (2009).

23 Cheung, H. O.-L. et al. The kinesin protein Kif7 is a critical regulator of Gli transcription factors in mammalian hedgehog signaling. Science signaling 2, ra29-ra29 (2009).

24 Endoh-Yamagami, S. et al. The mammalian Cos2 homolog Kif7 plays an essential role in modulating Hh signal transduction during development. Current biology 19, 1320-1326 (2009).

25 He, M. et al. The kinesin-4 protein Kif7 regulates mammalian Hedgehog signalling by organizing the cilium tip compartment. Nature cell biology 16, 663-672 (2014).

Maurya, A. K. et al. Positive and negative regulation of Gli activity by Kif7 in the zebrafish embryo. PLoS Genet 9, e1003955 (2013). signaling and microtubule dynamics. The Journal of clinical investigation 121, 2662-2667 (2011).

28 Putoux, A. et al. KIF7 mutations cause fetal hydrolethalus and acrocallosal syndromes. Nature genetics 43, 601-606 (2011).

824

Blasius, T. L. et al. Sequences in the stalk domain regulate autoinhibition and ciliary tip localization of the immotile kinesin-4 KIF7. Journal of Cell Science (2021).

30 Liu, Y. C. et al. The PPFIA1-PP2A protein complex promotes trafficking of Kif7 to the ciliary tip and Hedgehog signaling. Science signaling 7, ra117-ra117 (2014).

828 Schwarz, N. et al. Arl3 and RP2 regulate the trafficking of ciliary tip kinesins. Human molecular genetics 26, 2480-2492 (2017).

32 Ye, F., Nager, A. R. \& Nachury, M. V. BBSome trains remove activated GPCRs from cilia by enabling passage through the transition zone. Journal of Cell Biology 217, 1847-1868 (2018).

33 Eguether, T., Cordelieres, F. P. \& Pazour, G. J. Intraflagellar transport is deeply integrated in hedgehog signaling. Molecular biology of the cell 29, 1178-1189 (2018).

34 Pavletich, N. P. \& Pabo, C. O. Crystal structure of a five-finger GLI-DNA complex: new perspectives on zinc fingers. Science 261, 1701-1707 (1993).

35 Dan, S., Tanimura, A. \& Yoshida, M. Interaction of Gli2 with CREB protein on DNA elements in the long terminal repeat of human T-cell leukemia virus type 1 is responsible for transcriptional activation by tax protein. Journal of virology 73, 3258-3263 (1999).

36 Hoenger, A. et al. A new look at the microtubule binding patterns of dimeric kinesins. Journal of molecular biology 297, 1087-1103 (2000).

842

843

844

37 Hirose, K. et al. Structural comparison of dimeric Eg5, Neurospora kinesin (Nkin) and Ncd head-Nkin neck chimera with conventional kinesin. The EMBO Journal 19, 5308-5314 (2000).

845

38 Katoh, Y. \& Katoh, M. KIF27 is one of orthologs for Drosophila Costal-2. International journal of oncology 25, 1875-1880 (2004). 
39 Katoh, Y. \& Katoh, M. Characterization of KIF7 gene in silico. International journal of oncology 25, 1881-1886 (2004).

40 He, M., Agbu, S. \& Anderson, K. V. Microtubule motors drive hedgehog signaling in primary cilia. Trends in cell biology 27, 110-125 (2017).

41 Araghi, R. R. et al. Iterative optimization yields Mcl-1-targeting stapled peptides with selective cytotoxicity to Mcl-1-dependent cancer cells. Proceedings of the National Academy of Sciences 115, E886-E895 (2018).

42 Araghi, R. R. \& Keating, A. E. Designing helical peptide inhibitors of protein-protein interactions. Current opinion in structural biology 39, 27-38 (2016).

43 Foight, G. W., Chen, T. S., Richman, D. \& Keating, A. E. in Modeling Peptide-Protein Interactions 213-232 (Springer, 2017).

44 Truebestein, L. \& Leonard, T. A. Coiled-coils: The long and short of it. Bioessays 38, 903916 (2016).

45 Yüksel, D., Bianco, P. R. \& Kumar, K. De novo design of protein mimics of B-DNA. Molecular BioSystems 12, 169-177 (2016).

46 Tsonis, P. A. \& Dwivedi, B. Molecular mimicry: structural camouflage of proteins and nucleic acids. Biochimica et Biophysica Acta (BBA)-Molecular Cell Research 1783, 177187 (2008).

47 Wang, H. C., Chou, C. C., Hsu, K. C., Lee, C. H. \& Wang, A. H. J. New paradigm of functional regulation by DNA mimic proteins: recent updates. IUBMB life 71, 539-548 (2019).

48 Wang, H.-C., Ho, C.-H., Hsu, K.-C., Yang, J.-M. \& Wang, A. H.-J. DNA mimic proteins: functions, structures, and bioinformatic analysis. Biochemistry 53, 2865-2874 (2014).

49 Bochkareva, E. et al. Single-stranded DNA mimicry in the p53 transactivation domain interaction with replication protein A. Proceedings of the National Academy of Sciences 102, 15412-15417 (2005).

50 Liu, D. et al. Solution structure of a TBP-TAFII230 complex: protein mimicry of the minor groove surface of the TATA box unwound by TBP. Cell 94, 573-583 (1998).

51 Adriaans, I. E. et al. MKLP2 Is a Motile Kinesin that Transports the Chromosomal Passenger Complex during Anaphase. Current Biology (2020).

52 Ferry, L. et al. Methylation of DNA ligase 1 by G9a/GLP recruits UHRF1 to replicating DNA and regulates DNA methylation. Molecular cell 67, 550-565. e555 (2017).

53 Kino, T., Hurt, D. E., Ichijo, T., Nader, N. \& Chrousos, G. P. Noncoding RNA gas5 is a growth arrest-and starvation-associated repressor of the glucocorticoid receptor. Science signaling 3, ra8-ra8 (2010).

54 Liu, L., Yin, M., Wang, M. \& Wang, Y. Phage AcrIIA2 DNA mimicry: structural basis of the CRISPR and anti-CRISPR arms race. Molecular cell 73, 611-620. e613 (2019).

55 Kaan, H. Y. K., Hackney, D. D. \& Kozielski, F. The structure of the kinesin-1 motor-tail complex reveals the mechanism of autoinhibition. Science 333, 883-885 (2011).

56 Lee, P. L., Ohlson, M. B. \& Pfeffer, S. R. The Rab6-regulated KIF1C kinesin motor domain contributes to Golgi organization. Elife 4, e06029 (2015).

57 Kevenaar, J. T. et al. Kinesin-binding protein controls microtubule dynamics and cargo trafficking by regulating kinesin motor activity. Current Biology 26, 849-861 (2016).

58 Ren, J. et al. Coiled-coil 1-mediated fastening of the neck and motor domains for kinesin3 autoinhibition. Proceedings of the National Academy of Sciences 115, E11933-E11942 (2018). 
59 Wang, G. \& Jiang, J. Multiple Cos2/Ci interactions regulate $\mathrm{Ci}$ subcellular localization through microtubule dependent and independent mechanisms. Developmental biology 268, 493-505 (2004).

Zhou, Q. \& Kalderon, D. Costal 2 interactions with Cubitus interruptus (Ci) underlying Hedgehog-regulated Ci processing. Developmental biology 348, $47-57$ (2010).

61 Matise, M. P. \& Joyner, A. L. Gli genes in development and cancer. Oncogene 18, 78527859 (1999).

62 i Altaba, A. R., Sánchez, P. \& Dahmane, N. Gli and hedgehog in cancer: tumours, embryos and stem cells. Nature Reviews Cancer 2, 361-372 (2002).

63 Peer, E., Tesanovic, S. \& Aberger, F. Next-generation Hedgehog/GLI pathway inhibitors for cancer therapy. Cancers 11, 538 (2019).

904

64 Bird, L. E. et al. Green fluorescent protein-based expression screening of membrane proteins in Escherichia coli. Journal of visualized experiments: JoVE (2015).

905

Saeed, I. A. \& Ashraf, S. S. Denaturation studies reveal significant differences between GFP and blue fluorescent protein. International journal of biological macromolecules $\mathbf{4 5}$, 236-241 (2009).

66 Camacho, C. et al. BLAST+: architecture and applications. BMC bioinformatics 10, 421 (2009).

Remmert, M., Biegert, A., Hauser, A. \& Söding, J. HHblits: lightning-fast iterative protein sequence searching by HMM-HMM alignment. Nature methods 9, 173-175 (2012).

913 Guex, N., Peitsch, M. C. \& Schwede, T. Automated comparative protein structure modeling with SWISS-MODEL and Swiss-PdbViewer: A historical perspective. Electrophoresis 30, S162-S173 (2009). complexes. Nucleic acids research 46, W296-W303 (2018). Benkert, P., Biasini, M. \& Schwede, T. Toward the estimation of the absolute quality of individual protein structure models. Bioinformatics 27, 343-350 (2011).

71 Pettersen, E. F. et al. UCSF Chimera--a visualization system for exploratory research and analysis. J Comput Chem 25, 1605-1612, doi:10.1002/jcc.20084 (2004).

72 Jurrus, E. et al. Improvements to the APBS biomolecular solvation software suite. Protein Sci 27, 112-128, doi:10.1002/pro.3280 (2018).

73 Kozakov, D. et al. The ClusPro web server for protein-protein docking. Nature protocols 12, 255 (2017).

74 De Beer, T. A., Berka, K., Thornton, J. M. \& Laskowski, R. A. PDBsum additions. Nucleic acids research 42, D292-D296 (2014).

75 Suloway, C. et al. Automated molecular microscopy: the new Leginon system. $J$ Struct Biol 151, 41-60, doi:10.1016/j.jsb.2005.03.010 (2005).

76 Wilson-Kubalek, E. M., Cheeseman, I. M. \& Milligan, R. A. Structural comparison of the Caenorhabditis elegans and human Ndc80 complexes bound to microtubules reveals distinct binding behavior. Mol Biol Cell 27, 1197-1203, doi:10.1091/mbc.E15-12-0858 (2016).

932 processing. J Struct Biol 166, 95-102 (2009).

936

$78 \mathrm{Li}, \mathrm{X}$. et al. Electron counting and beam-induced motion correction enable near-atomicresolution single-particle cryo-EM. Nature methods 10, 584-590 (2013). 
93779 Hirschi, M. et al. Cryo-electron microscopy structure of the lysosomal calcium-permeable channel TRPML3. Nature 550, 411-414, doi:10.1038/nature24055 (2017).

80 Rohou, A. \& Grigorieff, N. CTFFIND4: Fast and accurate defocus estimation from electron micrographs. J Struct Biol 192, 216-221, doi:10.1016/j.jsb.2015.08.008 (2015).

81 Ogura, T., Iwasaki, K. \& Sato, C. Topology representing network enables highly accurate classification of protein images taken by cryo electron-microscope without masking. $J$ Struct Biol 143, 185-200 (2003).

82 Sui, H. \& Downing, K. H. Structural basis of interprotofilament interaction and lateral deformation of microtubules. Structure 18, 1022-1031, doi:10.1016/j.str.2010.05.010 (2010).

83 Egelman, E. H. The iterative helical real space reconstruction method: surmounting the problems posed by real polymers. $J$ Struct Biol 157, 83-94, doi:10.1016/j.jsb.2006.05.015 (2007).

84 Alushin, G. M. et al. High-resolution microtubule structures reveal the structural transitions in alphabeta-tubulin upon GTP hydrolysis. Cell 157, 1117-1129, doi:10.1016/j.cell.2014.03.053 (2014).

85 Tang, G. et al. EMAN2: an extensible image processing suite for electron microscopy. $J$ Struct Biol 157, 38-46, doi:10.1016/j.jsb.2006.05.009 (2007).

86 Grigorieff, N. FREALIGN: high-resolution refinement of single particle structures. $J$ Struct Biol 157, 117-125, doi:10.1016/j.jsb.2006.05.004 (2007). Afonine, P. V., Headd, J. J., Terwilliger, T. C. \& Adams, P. D. New tool: single microtubules by PRC1 and kinesin-4. Cell 154, 377-390 (2013). conserved nonmotor microtubule binding protein. Cell 142, 433-443 (2010). 


\section{Acknowledgements}

965 We thank Dr. Robert E. Kingston (Massachusetts General Hospital), Dr. Kathryn V. Anderson

966 (Sloan Kettering Institute), Dr. Robert Lipinski (University of Wisconsin), Dr. Adrian Salic

967 (Harvard Medical School), and Dr. Stephane Angers (University of Toronto) for kindly providing

968 us with the HeLa and MEF (WT, Gli2-/-, Gli2-/-Gli3-/-, Kif7-/-) cell lines. We also thank Dr. Mu

969 He (University of California San Francisco) for valuable discussions. This work was supported by

970 a grant to R.S. from American Cancer Society. R.S. is a Pew Biomedical Scholar and a recipient

971 of the Smith Family Award for Excellence in Biomedical Research.

972

\section{Author Contributions}

974 R.S., and F.H. designed the project. R.S., E.M.W.-K and R.A.M. designed the cryo-EM

975 experiments; F.H., performed pull-down assays, homology modelling, in vitro reconstitution,

976 TIRF microscopy, co-sedimentation and cell biological experiments; C.F. purified recombinant

977 proteins and performed stoichiometry and BLI assays; Q.Y. performed initial pull-down assays;

978 E.M.W.-K. performed cryo-EM experiments and obtained 3D reconstructions; N.M. did model

979 building in EM map; P.-I.K. performed cytoplasmic sequestration image analysis; F.H., C.F. and

980 R.S. wrote the manuscript. All authors discussed the results and reviewed the manuscript.

982 Extended data and Supplementary Information are available for this paper. 


\section{Figure 1}

a
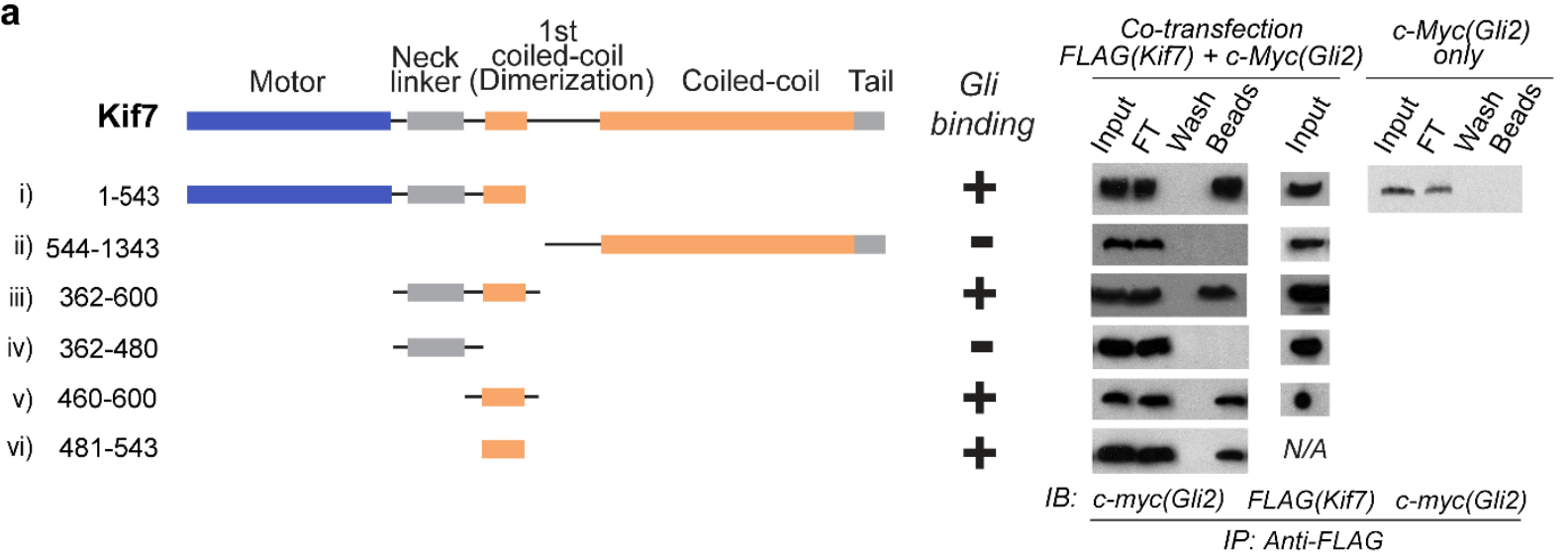

b
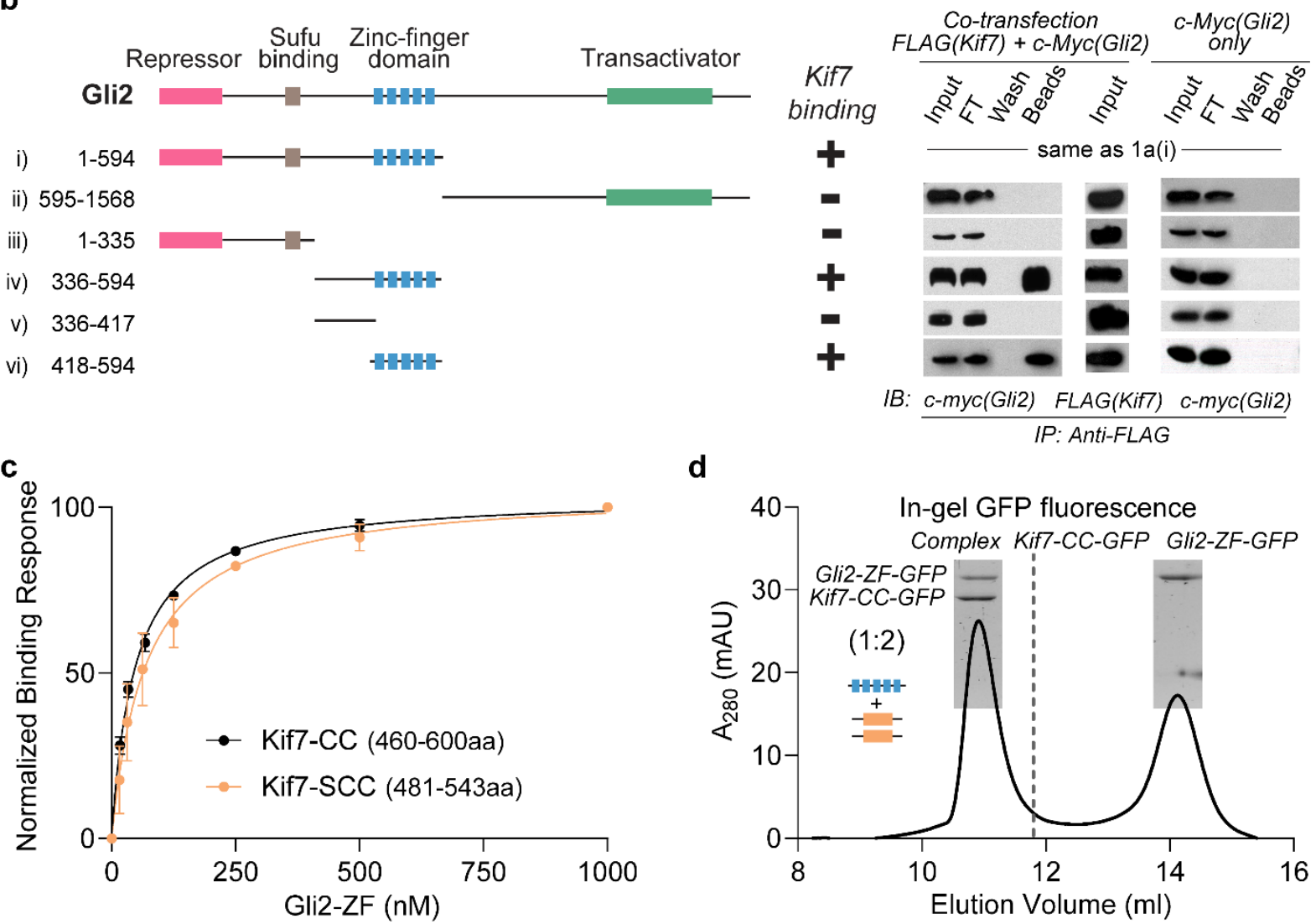

984 Figure 1 | DNA-binding domain of Gli2 forms a high affinity complex with the dimerization

985 domain of Kif7. a, Elucidation of the Kif7 domains that interact with Gli2. Pull-down of c-Myc-

986 Gli2(1-594aa) and FLAG-Kif7 constructs (i to vi) after co-transfection in Expi293F cells. (left

987 panel) Domain architecture of full length Kif7 and deletion constructs used in this experiment. 
988 (right panel) Immunoprecipitation (IP) using anti-FLAG magnetic beads. Input (cell lysate), FT

989 (flow through), wash and beads samples were immunoblotted (IB) with anti c-myc antibody to

990 detect Gli2. Transfection with c-Myc-Gli2 (1-594aa) alone was included as a negative control. The

991 blots are representative images of a minimum of three repeats. (center panel) Summary of results.

992 The +/- sign represents presence/absence of binding. b, Elucidation of the Gli2 domains that

993 interact with Kif7. Pull-down of c-Myc-Gli2 constructs (i to vi) with different FLAG-Kif7

994 constructs after co-transfection in Expi293F cells. Kif7(1-543aa) was used for i) \& ii), Kif7(362-

995 600aa) was used for iii) \& iv) and Kif7(460-600aa) was used for v) \& vi). (left panel) Domain

996 architecture of full length Gli2 and deletion constructs used in this experiment. (right panel)

997 Immunoprecipitation (IP) using anti-FLAG magnetic beads. Input (cell lysate), FT (flow through),

998 wash and beads samples were immunoblotted (IB) with anti c-myc antibody to detect Gli2.

999 Transfection with each of the different c-Myc-Gli2 constructs alone were included as negative

1000 controls. The blots are representative images of a minimum of three repeats. (center panel)

1001 Summary of results. The +/- sign represents presence/absence of binding. c, Bio-Layer

1002 Interferometry (BLI) assay to quantitatively examine the binding affinity of Kif7 coiled-coil

1003 domain constructs; GST-Kif7-CC (460-600aa; black) \& GST-Kif7-SCC (481-543aa; orange), to

1004 Gli2-ZF. Data represent mean and standard deviation from three independent repeats. The plots of

1005 binding response versus Gli2-ZF concentration were fit to a Hill equation to determine equilibrium

1006 dissociation constants $\left(\mathrm{K}_{\mathrm{d}}\right)$. For Kif7-CC (460-600aa): $\mathrm{K}_{\mathrm{d}}=48 \pm 5 \mathrm{nM}$, for Kif7-SCC (481-543aa):

$1007 \mathrm{~K}_{\mathrm{d}}=69 \pm 20 \mathrm{nM}$. d, Chromatograms from size exclusion chromatography of a mixture of $125 \mu \mathrm{M}$

1008 Kif7-CC-GFP and 750 $\mu$ M Gli2-ZF-GFP (Superdex 200 10/300 GL). SDS-PAGE of the complex

1009 (inset) was used to determine stoichiometry by in-gel GFP fluorescence analysis and is indicated

1010 in parenthesis. Gel image is representative of a minimum of three repeats. 
Figure 2

a

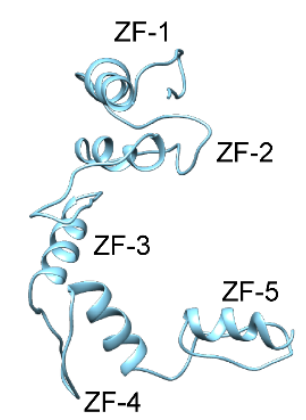

C

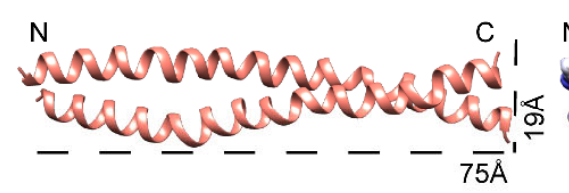

d

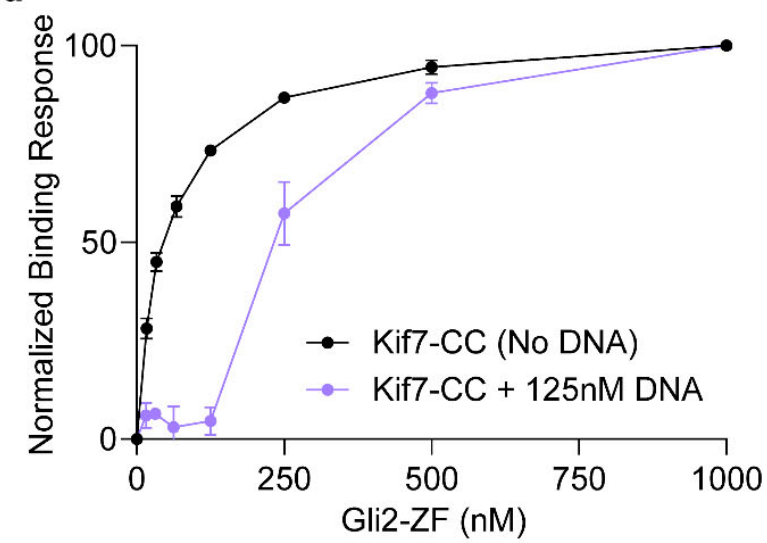

b

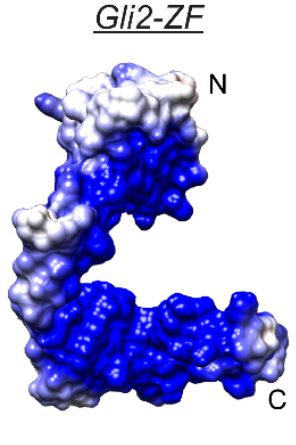

$\underline{K i f 7-C C}$

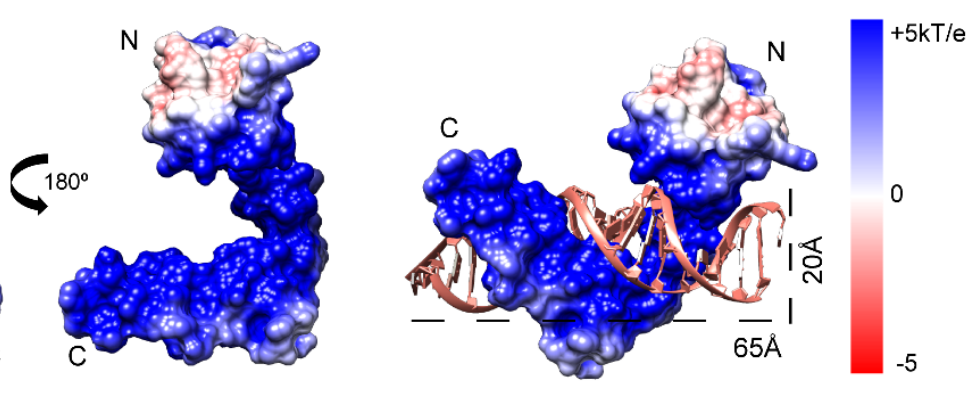

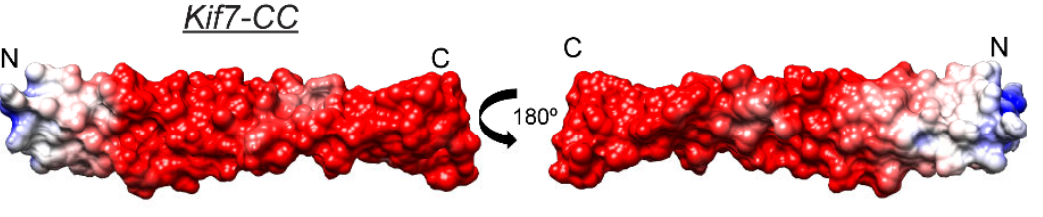

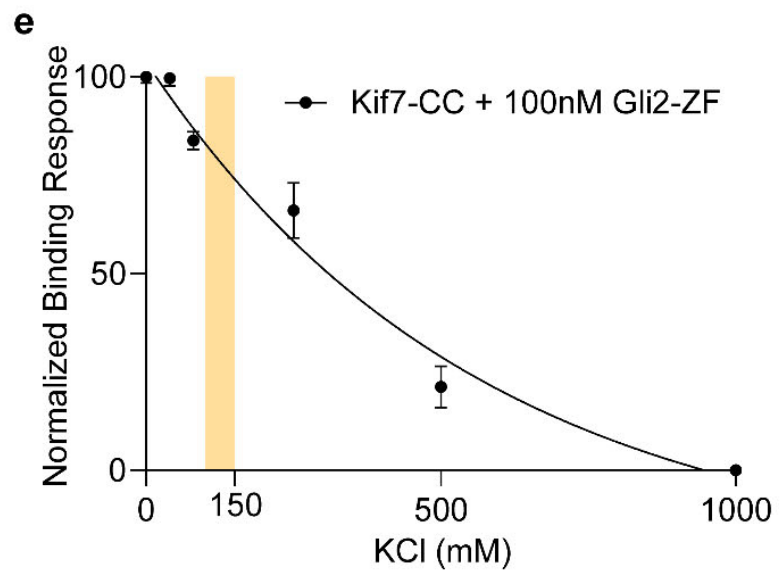

1012 Figure 2 | The coiled-coil dimerization domain of Kif7 is a DNA structural mimic for Gli

1013 binding. a, Structural model of Gli2-ZF (ribbon diagram; SWISS MODEL server: GMQE =0.98,

1014 QMean = -5.14) and overall electrostatic surface representation of the model. b, Structural model

1015 of Gli2-ZF bound to DNA (based on Gli1-ZF-DNA crystal structure; PDB: 2GLI) included for

1016 comparison. c, Structural model of Kif7-CC (ribbon diagram; SWISS MODEL server: GMQE =

10170.55 , QMean $=1.84)$ and overall electrostatic surface representation of the model. Dotted lines

1018 represent molecular dimensions of Kif7-CC and DNA. d, Binding of Gli2-ZF and Kif7-CC in the 1019 absence (black) and presence of $125 \mathrm{nM}$ sequence-specific Gli2 target dsDNA (purple). 
1020 Normalized binding response from the BLI measurements is plotted against Gli2-ZF

1021 concentration. Data represent mean and standard deviation from three independent repeats. e,

1022 Effect of increasing ionic strength ( $\mathrm{KCl}$ concentration) on the Kif7-CC and Gli2-ZF binding.

1023 Normalized binding response was measured at $100 \mathrm{nM}$ Gli2-ZF using the BLI assay. Data

1024 represent mean and standard deviation from three independent repeats. Yellow bar marks

1025 physiological ionic strength $(\sim 125-150 \mathrm{mM} \mathrm{KCl})$. 


\section{Figure 3}
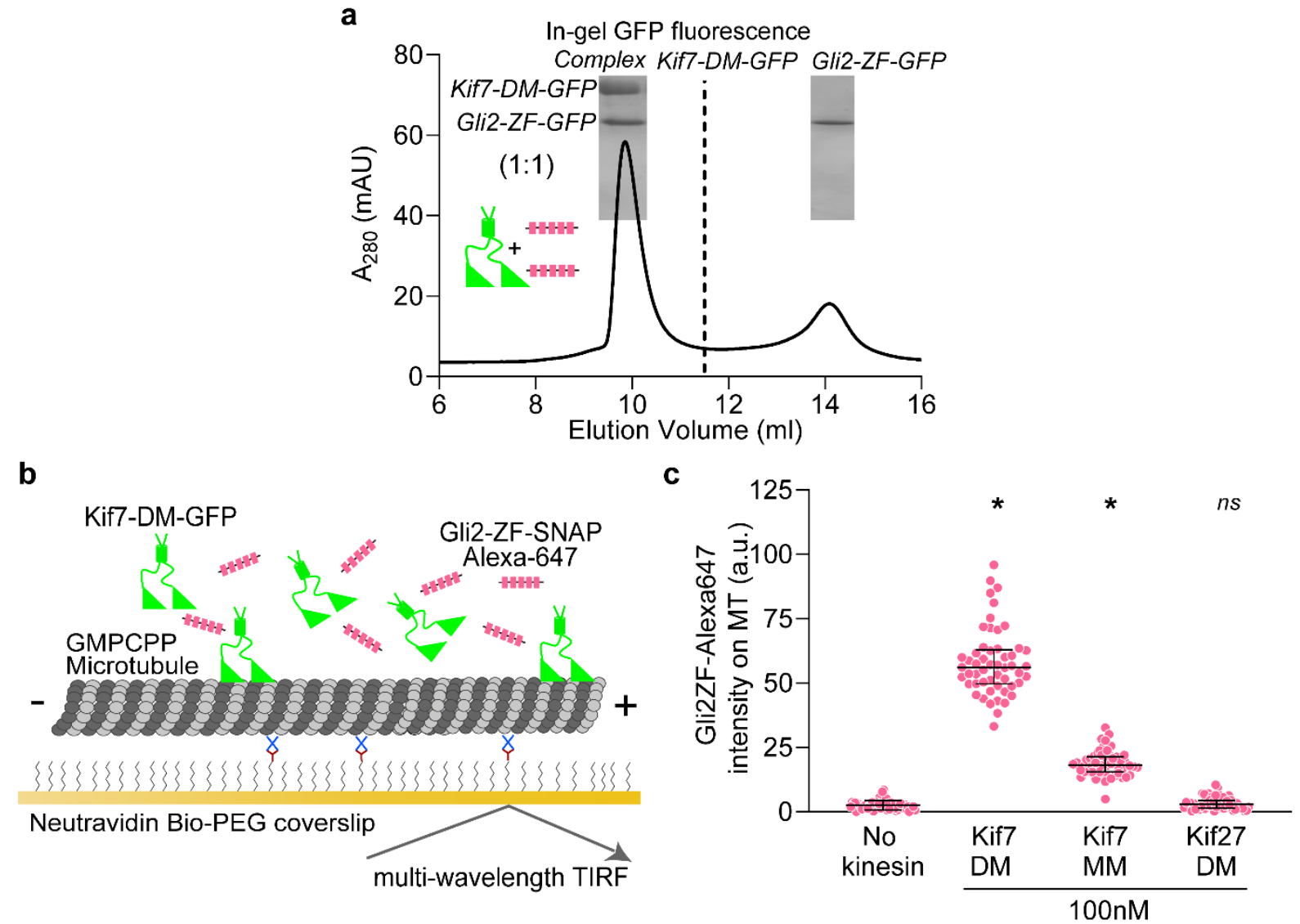

d

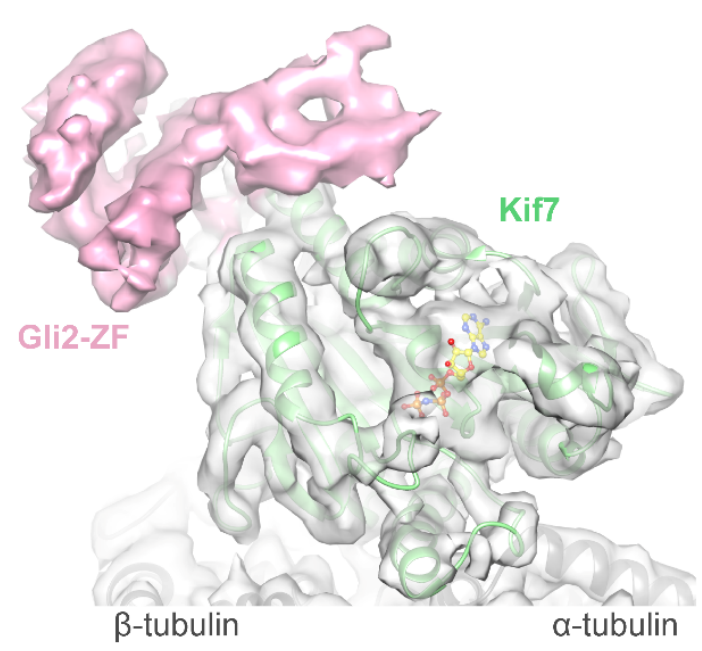

e
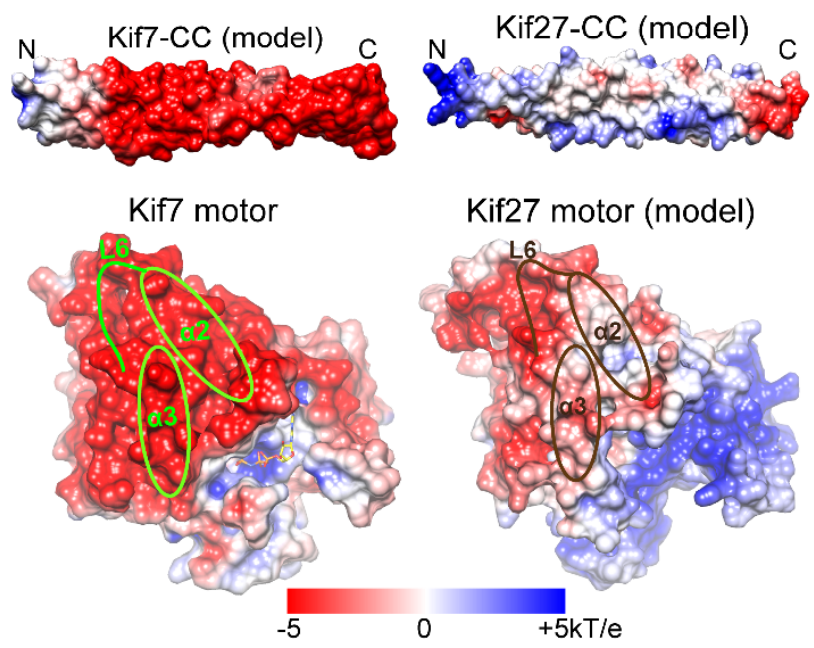

1027 Figure 3 | Second Gli interaction site on Kif7 motor domain. a, Chromatograms from size

1028 exclusion chromatography of a mixture of $125 \mu \mathrm{M}$ Kif7-DM-GFP and $750 \mu \mathrm{M}$ Gli2-ZF-GFP

1029 (Superdex 200 10/300 GL). SDS-PAGE of the complex (inset) was used to determine 
1030 stoichiometry by in-gel GFP fluorescence analysis and is indicated in parenthesis. Gel image is

1031 representative of a minimum of three repeats. $\mathbf{b}$, Schematic of the in vitro total internal reflection

1032 fluorescence (TIRF) microscopy-based assay used to examine microtubule-localized Gli2-ZF and

1033 Kif7-DM. Rhodamine/HiLyte 647 labeled GMPCPP-stabilized microtubules (grey) were

1034 immobilized on a PEG-treated glass coverslip (yellow) via neutravidin-biotin linkages (blue \&

1035 brown respectively). Kif7-DM-GFP (green), Gli2-ZF-SNAP-Alexa 647 (pink) and 1mM ATP

1036 were subsequently added to examine binding of both proteins on microtubules. c, Scatter plot of

1037 Gli2-ZF-Alexa647 intensity per pixel on microtubules (MT) represents recruitment of Gli on MT

1038 by Kif7-MM and Kif7-DM. The no kinesin condition was a control for non-specific binding of

1039 Gli2-ZF to MT and Kif27-DM was included as a negative control for Gli2-ZF binding. Assay

1040 conditions: $100 \mathrm{nM}$ Gli2-ZF-SNAP-Alexa647 with $100 \mathrm{nM}$ of kinesin in each case. $\mathrm{N} \geq 50$

1041 microtubules for each condition. One-way ANOVA $(p<0.0001)$ and post-hoc analysis $(* p<$

10420.0001 in Dunnett's multiple comparisons test) show statistically significant differences in Gli

1043 intensity compared to no kinesin control; $n s$ is not significant $(p=0.9253)$. d, Cryo-EM

1044 reconstruction of Kif7-DM bound to microtubules in the presence of Gli2-ZF-SNAP and

1045 AMPPNP. The structural model for Kif7 motor domain (green ribbon) bound to microtubule (grey

1046 ribbon) was fitted in the density and refined. The density corresponding to Gli2-SNAP is shown

1047 in pink. AMPPNP is shown as a yellow ball-and-stick model. e, Comparison of electrostatic

1048 surface potential of coiled-coil and motor domains of Kif7 (PDB: 6 MLR) and Kif27. The

1049 structural models of Kif7-CC (SWISS MODEL server: GMQE = 0.55, QMean = 1.84), Kif27-CC

1050 (SWISS MODEL server: GMQE $=0.51$, QMean $=0.84)$ and Kif27 motor domain (SWISS

1051 MODEL server: GMQE = 0.47, QMean = -2.22) were obtained by homology modelling. Loop L6,

1052 helices $\alpha-2$ and $\alpha-3$ on the Kif7 motor domain that lie close to the Gli2-SNAP density are 
bioRxiv preprint doi: https://doi.org/10.1101/2021.09.30.462597; this version posted October 1, 2021. The copyright holder for this preprint

(which was not certified by peer review) is the author/funder, who has granted bioRxiv a license to display the preprint in perpetuity. It is made available under aCC-BY-NC-ND 4.0 International license.

1053 highlighted in green. Corresponding regions (Loop L6, helices $\alpha-2$ and $\alpha-3$ ) in the Kif27 motor

1054 domain model are highlighted in brown. 
a
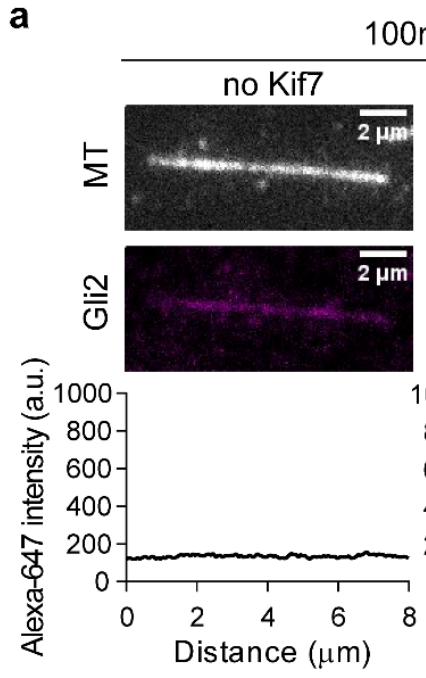

C

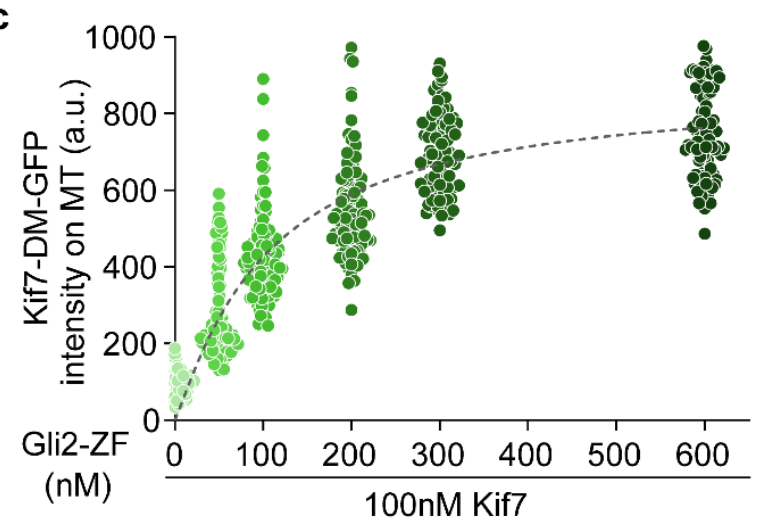

e

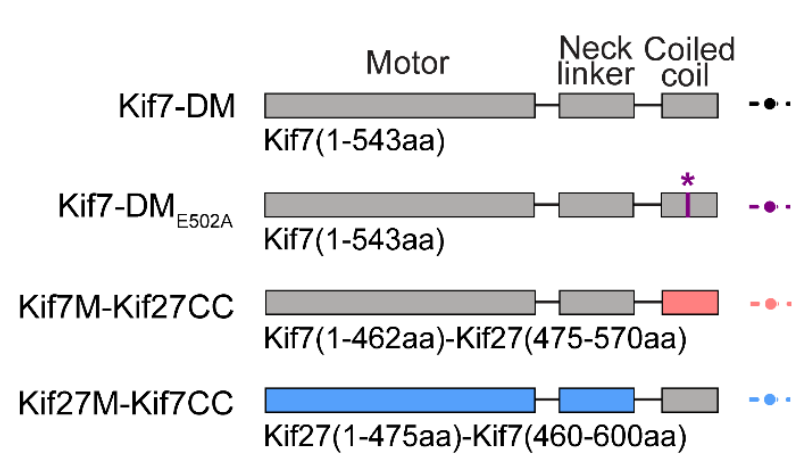

Figure 4

b

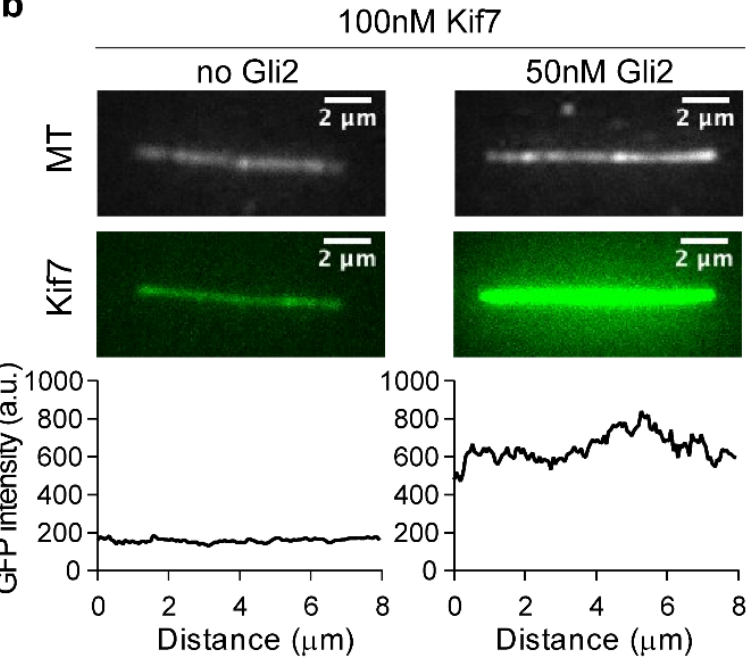

d $\overparen{0}$
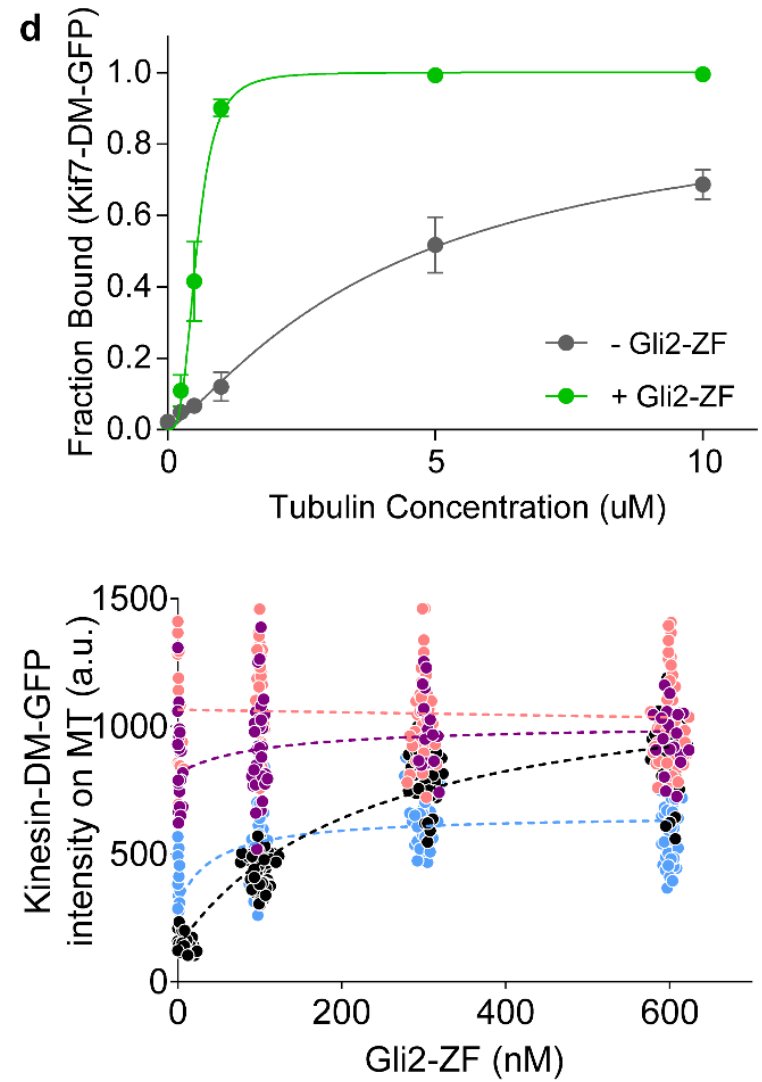

1056 Figure 4 | Gli2 ZF increases the microtubule-binding affinity of Kif7, thereby increasing its

1057 own recruitment on microtubules. a, Representative images of microtubule (MT, top) and Gli2-

1058 ZF-Alexa 647(Gli2, bottom, 100nM) in the absence (left) or presence of Kif7-DM-GFP (right,

$1059100 \mathrm{nM})$. The graphs below show line scans of the corresponding Alexa 647 fluorescence intensity. 
1060 Scale bars represent $2 \mu \mathrm{m}$. b, Representative images of microtubule (MT, top) and Kif7-DM-GFP

1061 (Kif7, bottom, 100nM) in the absence (left) or presence of Gli2-ZF (right, 50nM). The graphs

1062 below show line scans of the corresponding GFP fluorescence. Scale bars represent $2 \mu \mathrm{m}$. c, Scatter

1063 plot of Kif7-DM-GFP intensity per pixel on microtubules (MT) in the presence of increasing

1064 concentrations of Gli2-ZF. Assay conditions: 100nM Kif7-DM-GFP with 0, 50, 100, 200, 300 \&

1065 600nM Gli2-ZF. $\mathrm{N} \geq 90$ microtubules at every Gli2-ZF concentration. One-way ANOVA $(p<$

1066 0.0001) and post-hoc analysis $(p<0.0001$ in Dunnett's multiple comparisons test) shows

1067 statistically significant differences among means at various Gli2-ZF concentrations compared to

1068 no Gli2-ZF. Data fit to dose response curve (dotted grey line) with half maximal response at $\sim 160$

1069 nM Gli2-ZF. d, Co-sedimentation assay curve from analysis of gels in c, to quantitatively examine

1070 the microtubule binding affinity of Kif7DM-GFP in the absence (grey) and presence (blue) of

1071 Gli2-ZF. Error bars represent standard error. The plots of fraction of Kif7DM-GFP bound versus

1072 microtubule concentration were fit to a Hill equation to determine the equilibrium dissociation

1073 constants $\left(\mathrm{K}_{\mathrm{d}}\right)$. For Kif7-DM-GFP - Gli2-ZF: $\mathrm{K}_{d}=4.23 \pm 0.24 \mu \mathrm{M}\left(\mathrm{R}^{2}=0.93\right)$ and for Kif7-DM-

1074 GFP + Gli2-ZF: $K_{d}=0.54 \pm 0.03 \mu \mathrm{M}\left(\mathrm{R}^{2}=0.95\right)$. In the presence of Gli2-ZF, Kif7-DM-GFP

1075 shows co-operative binding to microtubules with a Hill co-efficient (h) of $3.2 \pm 0.59$. e, Schematics

1076 showing the domains swapped in the Kif7-Kif27 chimera proteins and the E502A point mutant

1077 Kif7-DM. Scatter plot of Kinesin-GFP intensity per pixel on microtubules (MT) in the presence

1078 of increasing concentrations of Gli2-ZF. Assay conditions: 100nM of each kinesin: Kif7-DM

1079 (black), Kif7-DMe502A (purple), Kif7M-Kif27CC (red) and Kif27M-Kif7CC (blue), with 0, 100,

$1080300 \& 600 \mathrm{nM}$ Gli2-ZF. $\mathrm{N} \geq 40$ microtubules for each data set. Two-way ANOVA (factor 1: Gli2-

1081 ZF concentration $p<0.0001$; factor 2: kinesin type $p<0.0001$ and their interaction $p<0.0001$ )

1082 and post-hoc analysis $(p<0.01$ in Dunnett's multiple comparisons test) shows statistically 
bioRxiv preprint doi: https://doi.org/10.1101/2021.09.30.462597; this version posted October 1, 2021. The copyright holder for this preprint (which was not certified by peer review) is the author/funder, who has granted bioRxiv a license to display the preprint in perpetuity. It is made available under aCC-BY-NC-ND 4.0 International license.

1083 significant differences among means at various Gli2-ZF concentrations compared to Kif7-DM.

1084 Data were fit to dose response curve (dotted lines). 
Figure 5

a

DAPI

Kif7-FL
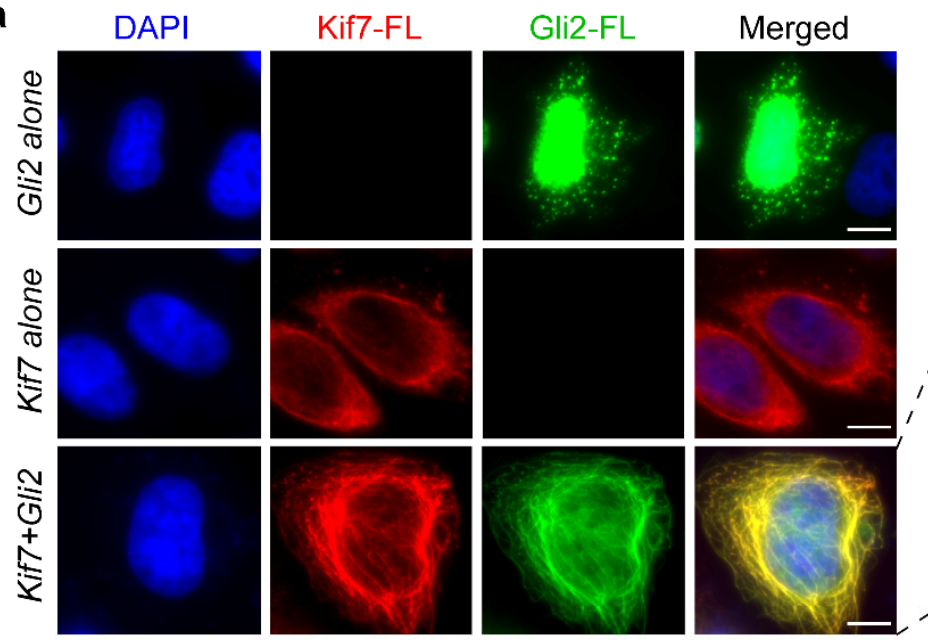

b
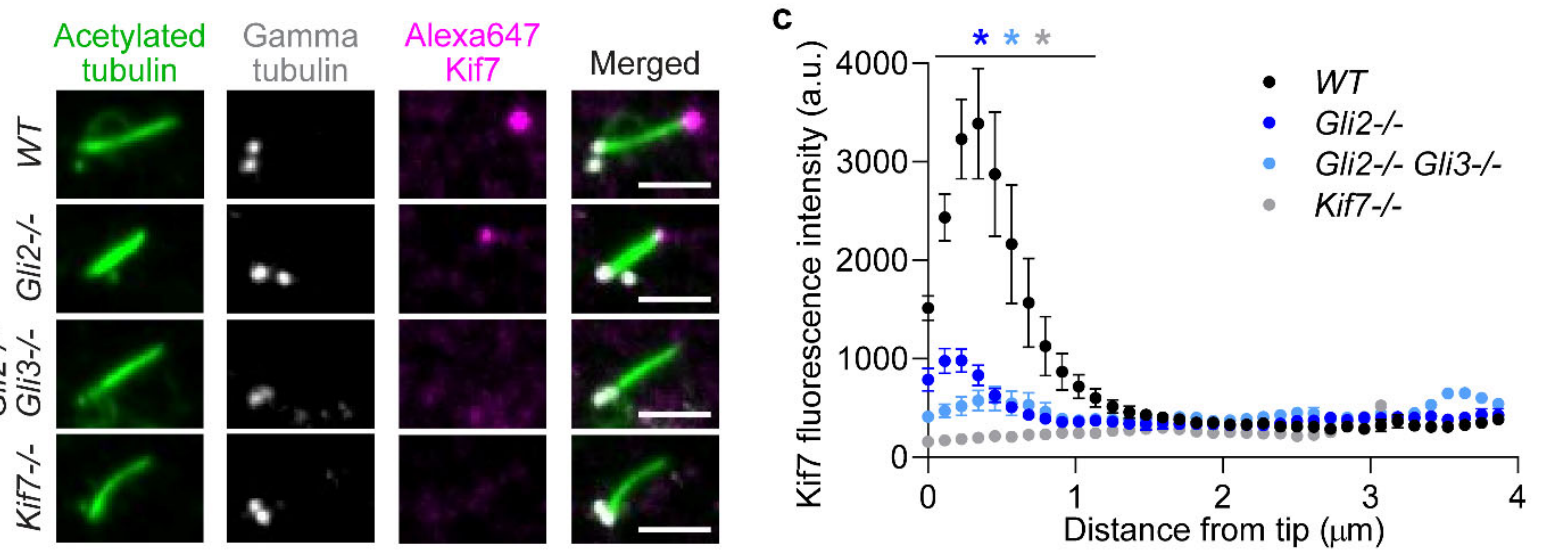

Figure 5 | Synergistic regulation of Gli-Kif7 promotes Kif7 localization to cytosolic

1087 microtubules and the distal cilium tip. a, Localization of transfected mRuby-tagged full length

1088 Kif7 and mNeonGreen-tagged full length Gli2 in HeLa cells. Representative images are shown.

1089 DAPI staining was used to mark the nucleus. Overexpressed full length Gli2 shows predominant

1090 localization in the nucleus (row 1). Overexpressed full length Kif7 is cytoplasmic (row 2). Co-

1091 transfection with Kif7 and Gli2 shows localization of both proteins on microtubules in the

1092 cytoplasm (row 3) $(100 \% ; \mathrm{n}>30)$. Scale bars represent $10 \mu \mathrm{m}$. b, Cilium-tip localization of Kif7 in

1093 WT, Gli2-/-, Gli2-/-Gli3-/- and Kif7-/- MEFs. Representative immunofluorescent images of

1094 primary cilia are shown. Acetylated $\alpha$-tubulin antibody was used to mark cilia, $\gamma$-tubulin antibody 
1095 was used to mark centrioles (base of cilia) and Alexa-Fluor-647-labeled Kif7 antibody was used

1096 to measure Kif7 amounts in the cilia tips during Hh activation (+SAG). Scale bar represents $2 \mu \mathrm{m}$.

1097 c, Quantitative analysis of the levels of Kif7 in WT, Gli2-/-, Gli2-/-Gli3-/- and Kif7-/- MEFs. Line

1098 scans of cilia from the experiment in (b), was used to quantify distribution of Kif7 along the length

1099 of the cilia. Data represent mean and standard error from three independent repeats $(\mathrm{N}=10$ cilia

1100 for each genotype). Two-way ANOVA (factor 1: genotype $p<0.0001$, factor 2: distance from tip

$1101 p<0.0001)$ and post-hoc analysis $\left({ }^{*} p<0.0001\right.$ in Dunnett's multiple comparison test $)$ show

1102 statistically significant differences among Kif7 intensity in cilia tip of all genotypes compared to

1103 wild type control. 
a
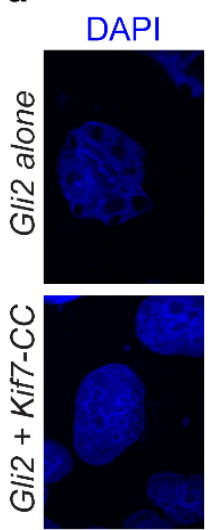

d
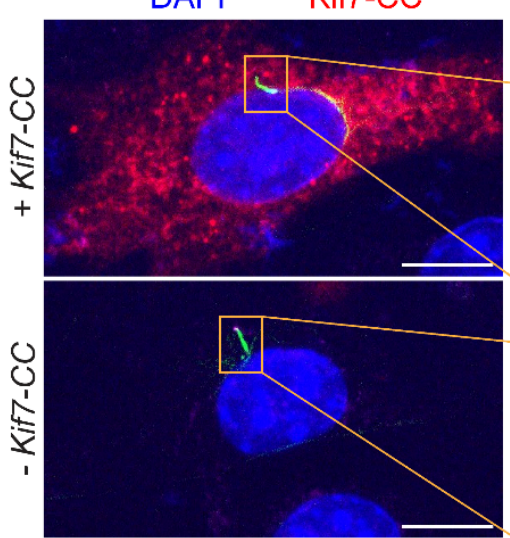
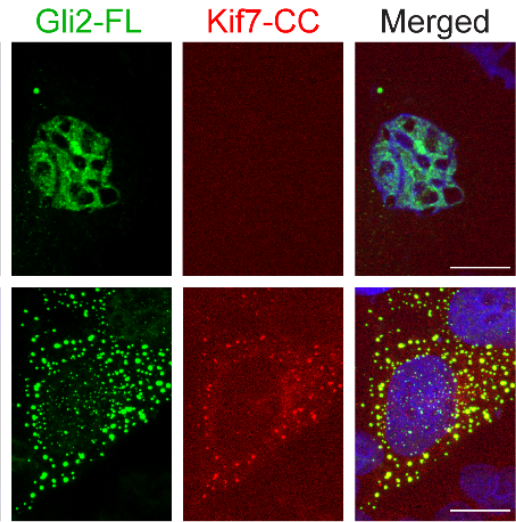

b

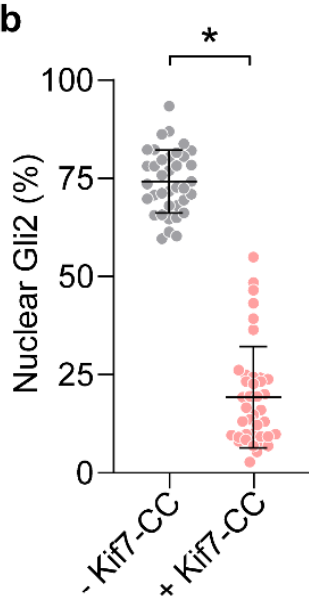

C

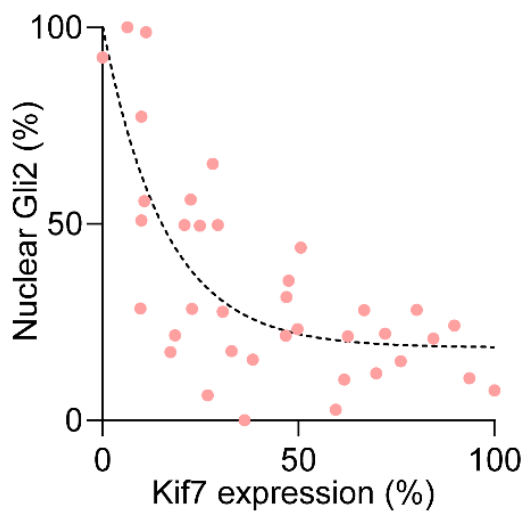

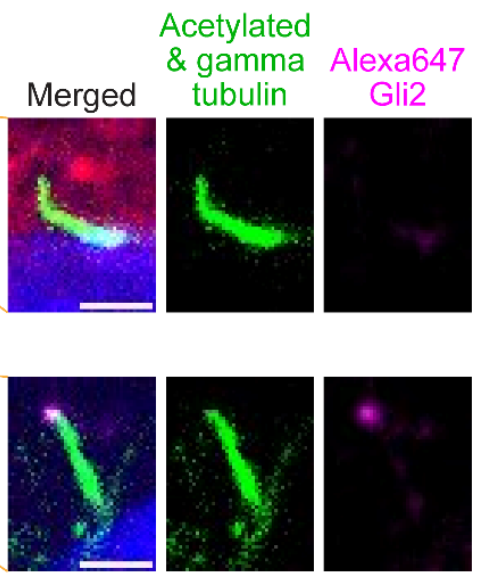

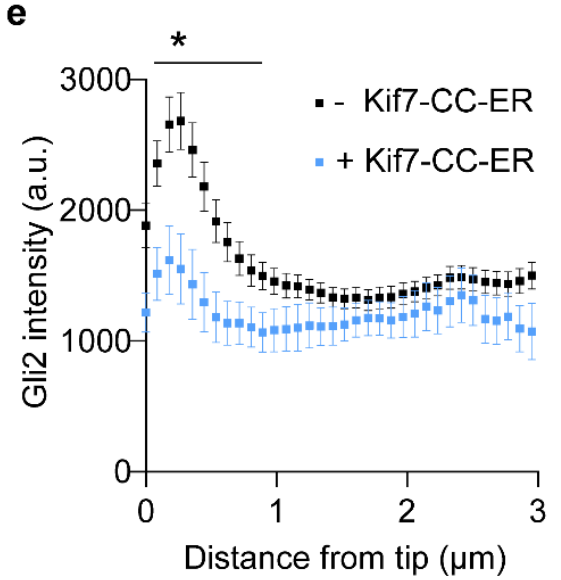

1105 Figure 6 | Re-engineering the DNA-mimicking coiled-coil domain of Kif7 as a tool for

1106 sequestration of Gli away from the nucleus and cilia. a, Localization of transfected mRuby-

1107 tagged Kif7-CC (460-600aa) with ER retention tag and mNeonGreen-tagged full length Gli2 in

1108 HeLa cells. Representative images are shown. DAPI staining was used to mark the nucleus.

1109 Overexpressed full length Gli2 shows predominant localization in the nucleus (row 1). Co-

1110 transfection with Kif7-CC and Gli2 shows co-localization of Gli2 in the cytoplasm with Kif7-CC

1111 (row 2). Scale bars represent $10 \mu \mathrm{m}$. b, Percent of nucleus-localized Gli2 as quantified from a, in

1112 the absence and presence of Kif7-CC. Data represent mean and standard deviation from three

1113 independent repeats ( $\mathrm{N} \geq 35$ for each condition). c, Scatter plot of percent nuclear Gli2 versus

1114 level of Kif7-CC expression. The dotted line represents fit of the data to one-phase association. d, 
1115 Representative immunofluorescent images of cilium-tip localization of endogenous Gli2 in

1116 NIH3T3 cell transfected with mRuby-tagged Kif7-CC (460-600aa) with ER retention tag (top row)

1117 and untransfected cell from the same sample (bottom row). Acetylated $\alpha$-tubulin antibody was

1118 used to mark cilia, $\gamma$-tubulin antibody was used to mark centrioles (base of cilia) and Alexa-Fluor-

1119 647-labeled Gli2 antibody was used to measure endogenous Gli2 amounts in the cilia tips during

1120 Hh activation (+SAG). Scale bar in the left panel represents $10 \mu \mathrm{m}$ and in the cilium inset represents

$11212 \mu \mathrm{m} . \mathbf{e}$, Quantitative analysis of the levels of Gli2 in cilia of NIH3T3 cells transfected with Kif7-

1122 CC-ER in the presence of SAG. Line scans of cilia from $\mathbf{d}$, was used to quantify distribution of

1123 Gli2 along the length of the cilia. Data represent mean and standard error from three independent

1124 repeats $(\mathrm{N} \geq 12$ cilia for each group). Two-way ANOVA (factor 1: transfected with Kif7-CC-ER

$1125 p<0.05$, factor 2: distance from tip $p<0.0001)$ and post-hoc analysis $(* p<0.0001$ in unpaired

1126 Student's t-test) shows statistically significant differences in Gli2 intensity in cilia tips upon Kif7-

1127 CC-ER transfection compared to non-transfected control. 


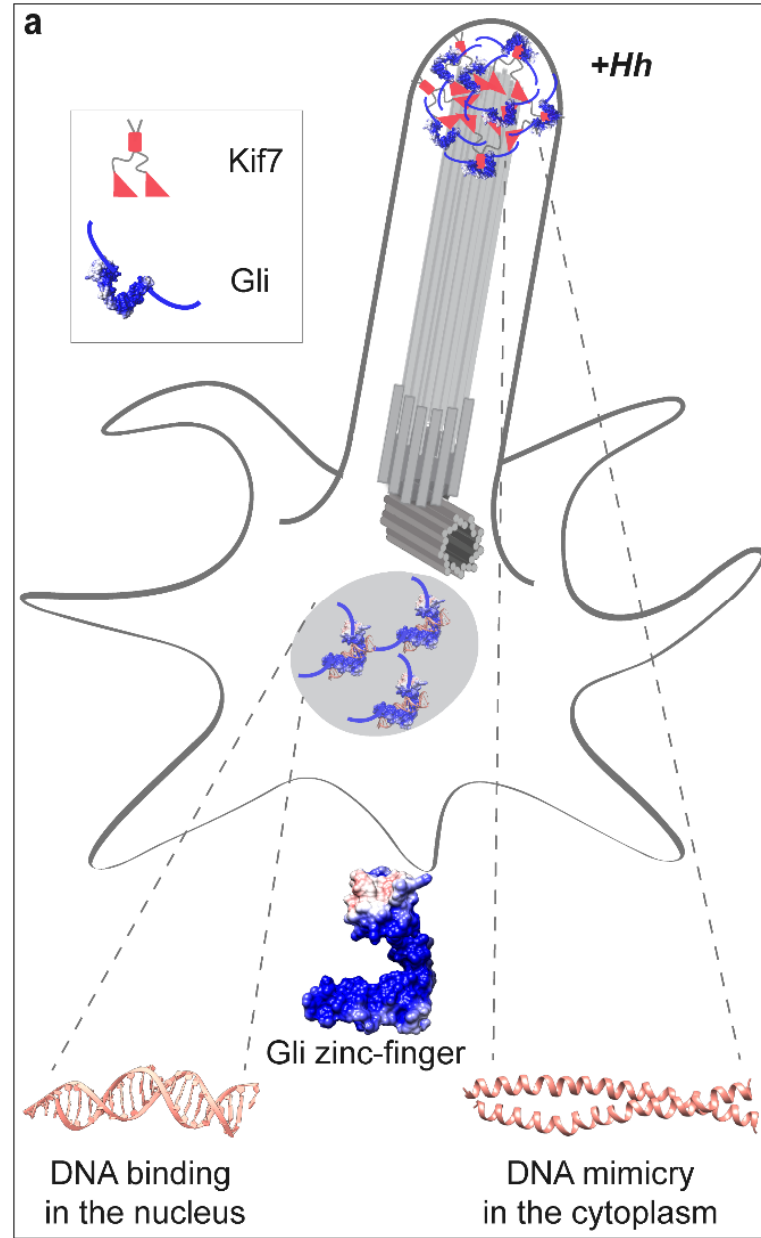

Figure 7

1129 Figure 7 | Schematic of the structural basis and cellular implications of Kif7-Gli interaction.

1130 a, DNA molecular mimicry by the Kif7 coiled-coil underlies the tethering of Gli in the cytoplasm

1131 and the cilia tips. The zinc-finger domain of Gli that binds DNA in the nucleus is co-opted for

1132 binding the Kif7 coiled-coil out of the nucleus. b, The Kif7 coiled-coil acts as a regulatory domain

1133 necessary for the graded accumulation of both proteins on microtubules and at the cilium tip in a

1134 Gli-concentration responsive manner. Consistent with this, Gli knockout cells (Gli-/-) have low

1135 levels of Kif7 at the cilia tips compared to WT cells during Hedgehog signal transduction. In Kif7

1136 knockout cells (Kif7-/-) Gli is distributed in puncta along the length of the ciliary axoneme and is

1137 not concentrated at the cilia tip during pathway activation ${ }^{25,30}$. c, The Kif7 coiled-coil peptide can 
bioRxiv preprint doi: https://doi.org/10.1101/2021.09.30.462597; this version posted October 1, 2021. The copyright holder for this preprint (which was not certified by peer review) is the author/funder, who has granted bioRxiv a license to display the preprint in perpetuity. It is made available under aCC-BY-NC-ND 4.0 International license.

1138 be re-tooled as a reagent for sequestration of Gli away in the cytoplasm to decrease nuclear and 1139 ciliary Gli levels. 